OPEN ACCESS

Edited by:

Jianhua Zhang,

University of Alabama at Birmingham,

United States

Reviewed by:

Martin Sebastian Denzel,

Max Planck Institute for Biology of

Ageing, Germany

Pablo Jose Fernandez-Marcos,

IMDEA Food Institute, Spain

*Correspondence:

Neil J. McKenna

nmckenna@bcm.edu

Qiang Tong

qtong@bcm.edu

Specialty section:

This article was submitted to Aging, Metabolism and Redox Biology,

a section of the journal

Frontiers in Aging

Received: 28 October 2021 Accepted: 13 December 2021

Published: 02 February 2022

Citation:

Chen KY, De Angulo A, Guo X, More A, Ochsner SA, Lopez E, Saul D, Pang W,

Sun Y, McKenna NJ and Tong $Q$ (2022) Adipocyte-Specific Ablation of

PU.1 Promotes Energy Expenditure and Ameliorates Metabolic Syndrome

in Aging Mice.

Front. Aging 2:803482

doi: 10.3389/fragi.2021.803482

\section{Adipocyte-Specific Ablation of PU.1 Promotes Energy Expenditure and Ameliorates Metabolic Syndrome in Aging Mice}

\author{
Ke Yun Chen ${ }^{1}$, Alejandra De Angulo ${ }^{1}$, Xin Guo ${ }^{1,2}$, Aditya More ${ }^{1}$, Scott A. Ochsner ${ }^{3}$, \\ Eduardo Lopez ${ }^{1}$, David Saul ${ }^{1}$, Weijun Pang ${ }^{1,4}$, Yuxiang Sun ${ }^{1,5}$, Neil J. McKenna ${ }^{3 *}$ and \\ Qiang Tong $1,6,7 *$
}

${ }^{1}$ Department of Pediatrics, USDA/ARS Children's Nutrition Research Center, Baylor College of Medicine, Houston, TX, United States, ${ }^{2}$ Department of Nutrition and Food Hygiene, School of Public Health, Cheeloo College of Medicine, Shandong University, Jinan, China, ${ }^{3}$ Department of Molecular and Cellular Biology, Baylor College of Medicine, Houston, TX, United States, ${ }^{4}$ Northwestern University of Agriculture and Forestry, Yangling, China, ${ }^{5}$ Department of Nutrition, Texas A\&M University, College Station, TX, United States, ${ }^{6}$ Department of Molecular Physiology and Biophysics, Baylor College of Medicine, Huffington Center on Aging, Houston, TX, United States, ${ }^{7}$ Department of Medicine, Baylor College of Medicine, Huffington Center on Aging, Houston, TX, United States

Objective: Although PU.1/Spi1 is known as a master regulator for macrophage development and function, we have reported previously that it is also expressed in adipocytes and is transcriptionally induced in obesity. Here, we investigated the role of adipocyte PU.1 in the development of the age-associated metabolic syndrome.

Methods: We generated mice with adipocyte-specific PU.1 knockout, assessed metabolic changes in young and older adult PU.1 $1^{\mathrm{fl} / \mathrm{fl}}$ (control) and AdipoqCre PU. $1^{\mathrm{fl} / \mathrm{fl}}$ (aPU.1KO) mice, including body weight, body composition, energy expenditure, and glucose homeostasis. We also performed transcriptional analyses using RNA-Sequencing of adipocytes from these mice.

Results: aPU.1KO mice have elevated energy expenditure at a young age and decreased adiposity and increased insulin sensitivity in later life. Corroborating these observations, transcriptional network analysis indicated the existence of validated, adipocyte PU.1modulated regulatory hubs that direct inflammatory and thermogenic gene expression programs.

Conclusion: Our data provide evidence for a previously uncharacterized role of PU.1 in the development of age-associated obesity and insulin resistance.

Keywords: Spi1/PU.1, thermogenesis, energy expenditure, inflammation, insulin resistance, obesity, aging, adipocyte

Abbreviations: Adipoq, adiponectin; GTT, glucose tolerance test; HCT, high confidence transcriptional targets; ITT, insulin tolerance test; LPS, lipopolysaccharide; MPO, Mammalian Phenotype Ontology; PGC-1, PPARG coactivator 1; RPA, Reactome Pathway Analysis; SPP, Signaling Pathways Project. 


\section{INTRODUCTION}

Systemic insulin resistance is a major global public health concern. Although it impacts numerous metabolic organs including skeletal muscle, liver and adipose tissue, evidence indicates that adipose tissue is one of the primary origins of systemic insulin resistance. It is well documented, for example, that obesity induces chronic low-grade inflammation in adipose tissue, a key event leading to systemic insulin resistance and metabolic syndrome (Hotamisligil, 2017). During obesity, adipose tissue secretes elevated levels of pro-inflammatory cytokines, such as TNF- $\alpha$, IL- $1 \beta$, IL- 6 and MCP-1 (Gregor and Hotamisligil, 2011). Obesity-associated adipose inflammation, for instance, is characterized by increased infiltration of macrophages (Weisberg et al., 2003; $\mathrm{Xu}$ et al., 2003) and other immune cells (Liu et al., 2009; Nishimura et al., 2009; Winer et al., 2009; Ohmura et al., 2010), and macrophage infiltration has been shown to be stimulated by obese adipose tissue expression of Ccl2/MCP-1 (Kamei et al., 2006; Weisberg et al., 2006). Although the role of macrophages is well-established in the inflammatory processes accompanying insulin resistance in adipose tissue, accumulating evidence implicates adipocytes as active participants in these processes.

PU.1 (encoded by Spi1) is a member of the ETS family of transcription factors (Klemsz et al., 1990) with historically well-characterized roles in the development of myeloid and lymphoid lineages, in particular macrophages and granulocytes (Scott et al., 1994; McKercher et al., 1996; Iwasaki et al., 2005). Functions for PU1.1 have also been established in lineage establishment of microglia (Kierdorf et al., 2013), dendritic cells (Carotta et al., 2010), and osteoclasts (Tondravi et al., 1997). Additionally, numerous lines of evidence cast macrophage PU.1 in a central role in the coordination of inflammatory transcriptional programs in macrophages. For example, macrophages lacking PU.1 are deficient in lipopolysaccharide (LPS) induction of Tlr4, Ptgs2 (encoding COX-2), Tnf, Illb, Il6, and Ccl2/MCP-1 (Karpurapu et al., 2011). In contrast to the volume of studies on PU.1 function in macrophages however, relatively little is known about its role in adipocytes. We previously reported that PU.1 was expressed in adipocytes, and that adipose tissue PU.1 expression was greatly increased in mouse models of obesity (Wang and Tong, 2008). Consistent with its pro-inflammatory role in macrophages, we found that depletion of PU.1 in cultured adipocytes led to decreased reactive oxygen species (ROS) production, increased insulin sensitivity, and reduced expression of signature obese adipose tissue cytokines, including TNF- $\alpha$, IL- $1 \beta$, and IL- $6{ }^{20}$. In addition, Lackey and colleagues have recently reported that mice with adipocyte-specific PU.1 knockout showed improved insulin sensitivity on a high fat diet, without any difference in body weight (Lackey et al., 2019).

The age-associated metabolic syndrome may have characteristics different from that caused by diet-induced obesity (Bapat et al., 2015). Here, we investigated the adipocyte-specific functions of PU.1 in mice, particularly during aging. We found that male mice with adipocyte-specific ablation of PU.1 had elevated energy expenditure, and were protected against age-associated obesity, with increased insulin sensitivity and increased glucose tolerance. Mechanistically, we performed validated informatics analyses that connect PU.1modulated transcriptional hubs to the observed physiological changes.

\section{EXPERIMENTAL PROCEDURES}

\section{Animals Experiments}

PU. $1^{\mathrm{f} / /+}$ mice, containing loxP sites flanking exon 5 of the Spi1 gene, were obtained from Dr. Stephen Nutts (Polli et al., 2005). These mice are on C57BL/ 6 genetic background and were used successfully for tissue specific knockout. Mice with adipocytespecific knockout of PU.1 (aPU.1KO) were generated by crossing Adiponectin-Cre mice (Adipoq-Cre, The Jackson Laboratory) (Eguchi et al., 2011) with PU.1 floxed mice. Mice were housed with littermates in cages and were fed on a standard chow diet (Lab Diet 5053; Purina Mills). All procedures used in animal experiments were approved by the Institution of Animal Care and Use Committee at Baylor College of Medicine. Mice were maintained under conditions of controlled temperature $\left(\sim 75^{\circ} \mathrm{F}\right)$ and illumination (12-hour light/12-hour dark cycle, 6 am to $6 \mathrm{pm}$ ) with free access to water.

\section{Glucose Tolerance Test (GTT) and Insulin Tolerance Test (ITT)}

Mice were fasted for overnight (GTT) or $4 \mathrm{~h}$ (ITT) and received an intraperitoneal injection of D-glucose $(2 \mathrm{~g} / \mathrm{kg})$ or insulin $(1 \mathrm{IU} / \mathrm{kg})$. Blood glucose was measured by glucose meter (TrueTest Glucose Meter and Strips) from the tail vein before and at 15, 30, 60, and $120 \mathrm{~min}$ after the bolus glucose or insulin injection.

\section{Body Composition Measurement and Calorimetry Experiment}

Mice body composition was measured using the EchoMRI-100 ${ }^{\mathrm{TM}}$ quantitative NMR instrument (Echo Medical Systems).

\section{Indirect Calorimetry}

Indirect calorimetry was measured using a computer-controlled, open-circuit system (Oxymax System) as part of an integrated Comprehensive Lab Animal Monitoring System (CLAMS; Columbus Instruments, Columbus, $\mathrm{OH}$, United States). Mice were singly housed in individual cages in adaptation for 3 days, followed by measurement for 4 days. On the last day, food was removed, and mice were fasted for $6 \mathrm{~h}$. Oxygen consumption (VO2) and carbon dioxide production (VCO2) were measured for each chamber and calculated by Oxymax software (v. 5.9). Energy expenditure was calculated as $\mathrm{EE}=3.815 \times \mathrm{VO} 2+1.232 \times$ $\mathrm{VCO} 2$. The basal energy expenditure was calculated based on the three lowest EE time points during the fasting period. 


\section{Adipose Tissue Fractionation}

Epididymal fat pads from mice were minced in Krebs-Ringer phosphate buffer and digested with $1 \mathrm{mg} / \mathrm{ml}$ collagenase type I (Worthington Biochemical) at $37^{\circ} \mathrm{C}$ for $1 \mathrm{~h}$ as described in the literature (Wang and Tong, 2008). Digested tissue was filtered through a nylon mesh and centrifuged at $500 \mathrm{rpm}$ for $10 \mathrm{~min}$. The top layer (adipocyte fraction) was collected. Proteins were extracted from adipocyte fraction for Western blot analysis. RNA was also prepared from adipocyte fraction for RNAsequencing and Real-time PCR analyses.

\section{RNA-Sequencing}

Epididymal adipocytes RNA were extracted from isolated adipocyte fraction of three control and three aPU.1KO mice using the RNeasy Lipid Tissue Mini Kit (QIAGEN). The cells were homogenized in $1 \mathrm{ml}$ QIAzol lysis reagent and centrifuged at $12,000 \mathrm{~g}$ for $10 \mathrm{~min}$ at $4^{\circ} \mathrm{C}$. The lysates under the lipid layer were transferred to a fresh tube and extracted with $200 \mu \mathrm{l}$ chloroform, centrifuged at $12,000 \mathrm{~g}$ for $15 \mathrm{~min}$ at $4^{\circ} \mathrm{C}$. The upper aqueous phase was transferred out and mixed with 1 volume of $70 \%$ ethanol. The samples were then applied to RNeasy Mini spin column and centrifuged at room temperature for $15 \mathrm{~s}$ at 8,000 g. The columns were washed once with $700 \mu \mathrm{L}$ Buffer RW1, and twice with $500 \mu \mathrm{L}$ Buffer RPE. The RNA samples were eluded with $30-50 \mu \mathrm{L}$ RNase-free water. RNA-sequencing was performed by Novogene Corporation Inc. (Sacramento, CA).

\section{RNA-Seq Analysis}

Sequencing was performed on adipocyte samples from PU.1 $1^{\mathrm{f} / \mathrm{fl}}$ (control) and PU.1 $1^{\mathrm{fl} / \mathrm{fl}}$-AdipoqCre (PU.1 knockout) mice, with three replicates in each group. Sequencing reads were quantified using Salmon with the option-validateMappings for a more sensitive mapping scheme (Patro et al., 2017). Transcript-level counts were summed to the gene-level for differential expression analysis using DESeq2 (Love et al., 2014).

\section{Real-Time PCR}

Adipocytes were isolated from mice gonadal adipose tissue. Total RNA of adipocytes was isolated using TRIzol Reagent (Invitrogen, Carlsbad, CA) following the manufacturer's instructions. The cDNA was synthesized using the SuperScript III First-Strand Synthesis System for RT-PCR (Invitrogen, Carlsbad, CA). qRT-PCR reactions were performed using iTaq Universal SYBR Green in a CFX96 Touch Real-Time PCR Detection System (Bio-Rad). The $\Delta \mathrm{Ct}$ method $(2-\Delta \mathrm{Ct})$ was used to calculate the relative mRNA expression level of each gene. Specific gene expression was normalized to $18 \mathrm{~S}$ ribosomal RNA. Sequences of primers used for real-time PCR were as follows: MCP-1-F 5'-GAAGGAATGGGTCCAGACAT- $3^{\prime}$ and MCP-1-R 5'-ACGGGTCAACTTCACATTCA-3'; TNFa-F $5^{\prime}$ ACGGGTCAACTTCACATTCA- $3^{\prime}$ and TNFa-R $5^{\prime}$-CTGATG AGAGGGAGGCCATT-3'; UCP-1-R $5^{\prime}$-AGCCACCACAGA AAGCTTGTCAAC- $3^{\prime}$ and UCP-1-R $5^{\prime}$-ACAGCTTGGTAC GCTTGGGTACTG-3'; PGC1 $\alpha-\mathrm{F} 5^{\prime}$ - GTCAACAGCAAAAGC CACAA- ${ }^{\prime}$ and PCG1 $\alpha-\mathrm{R} 5^{\prime}$-TCTGGGGTCAGAGGAAGAGA$3^{\prime}$; 18S ribosomal RNA-F 5' -AACGAGACTCTGGCATGCTAA CTAG- $3^{\prime}$ and $18 \mathrm{~S}$ ribosomal RNA-R $5^{\prime}$-CGCCACTTGTCCCTC
TAAGAA-3'. The expression levels of genes of interest were normalized by the levels of $18 \mathrm{~S}$ RNA.

\section{Western Blot Analysis}

Cells were lysed in lysis buffer ( $50 \mathrm{mM}$ Tris, $50 \mathrm{mM} \mathrm{KCl,} 20 \mathrm{mM}$ $\mathrm{NaF}, 1 \mathrm{mM} \mathrm{Na} \mathrm{VO}_{4}, 10 \mathrm{mM}$ EDTA, $1 \%$ NP-40, $1 \mathrm{mM}$ PMSF, $5 \mu \mathrm{g} / \mathrm{ml}$ leupeptin, $\mathrm{pH}$ 8.0). Protein concentration was determined with BCA protein assay kit (Pierce, Rockford, IL). Twenty microgram proteins of each sample were separated by SDS-PAGE and electro-transferred to nitrocellulose membrane for immunoblot analysis. The following antibodies were used: anti-PU.1 (Santa Cruz Biotechnology, Santa Cruz, CA; sc-352, 1: 500), anti-a-tubulin (Sigma, St. Louis, MO; T5168, 1:100,000), HRP-conjugated anti-mouse (Bio-Rad, Richmond, CA; 170-6,516, 1:30,000), anti-rabbit (Bio-Rad, 170-6,515, 1: 30,000. The SuperSignal West Pico Chemiluminescent kit (Pierce, Rockford, IL) was used as substrates.

\section{T3-L1 Adipogenesis Consensome}

Full details of the methods and principles underlying consensome analysis can be found in the original publication (Ochsner et al., 2019). Briefly, five transcriptomic datasets (GSE2192, GSE60745, GSE14004, GSE12929, GSE20696) generated from 3T3-L1 cells treated with a standard adipogenic cocktail were organized into appropriate contrasts comparing gene expression levels at different time points to day 0 expression levels. These contrasts were then processed by the consensome pipeline implemented in $\mathrm{R}$ as previously described. For each transcript, the algorithm counts the number of experiments where the significance for differential expression is $<0.05$, then generates the binomial probability, referred to as the consensome $p$-value (CPV), of observing that many or more nominally significant experiments out of the number of experiments in which the transcript was assayed, given a true probability of 0.05 . Genes were ranked firstly by $\mathrm{CPV}$, then by geometric mean fold change (GMFC). The 3T3-L1 adipogenesis consensome was validated against the GSEA adipogenesis Hallmark gene set using a hypergeometric test implemented in $\mathrm{R}$ as described in the results. The consensome analysis code has been deposited in the SPP GitHub account at https:/github.com/signalingpathways-project/ominer/.

\section{High Confidence Transcriptional Target Intersection Analysis}

Node and node family consensomes are gene lists ranked according to measures of the strength of their regulatory relationship with upstream signaling pathway nodes derived from independent publicly archived transcriptomic or ChIPSeq datasets. In the case of ChIP-Seq datasets, the strength of the regulatory relationship is inferred from the mean ChIP-Atlas (Oki et al., 2018) MACS2 peak strength across available archived ChIP-Seq datasets in which a given pathway node is the IP antigen. In the case of transcriptomic datasets, the strength of the regulatory relationship is inferred from the frequency of significant differential expression of a given gene across independent experiments involving perturbation of a member 


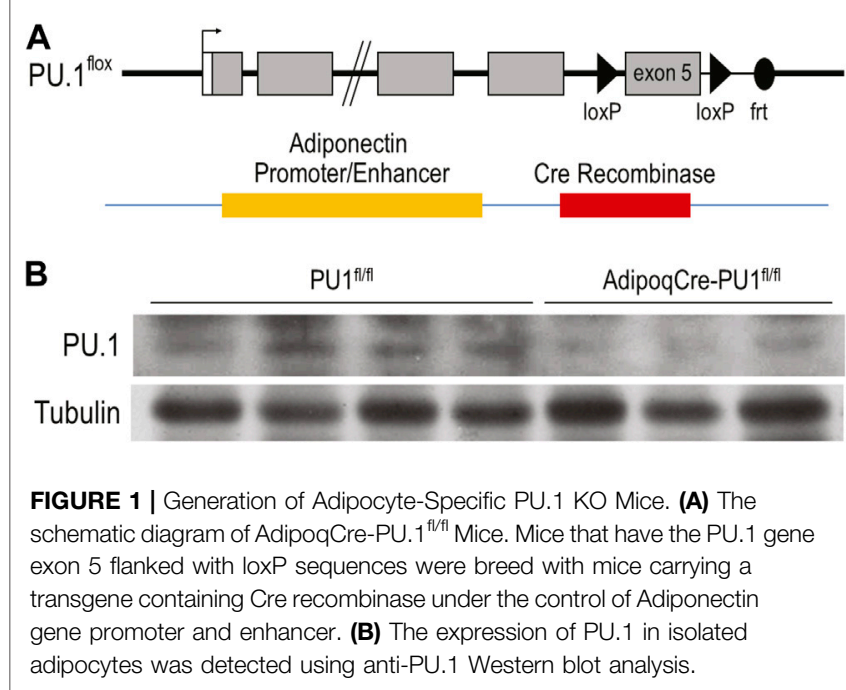

of a given node family (Ochsner et al., 2019). Genes in the 95th percentile of a given node consensome were designated high confidence transcriptional targets (HCTs) for that node and used as the input for the HCT intersection analysis using the Bioconductor GeneOverlap analysis package implemented in $\mathrm{R}$ as previously described (Ochsner et al., 2020). For both consensome and HCT intersection analysis, $p$ values were adjusted for multiple testing by using the method of Benjamini and Hochberg to control the false discovery rate as implemented with the p. adjust function in $\mathrm{R}$, to generate $Q$ values. Evidence for a transcriptional regulatory relationship between a node and a gene set was inferred from a larger intersection between the gene set and HCTs for a given node or node family than would be expected by chance after FDR correction $(\mathrm{Q}<0.05)$. The HCT intersection analysis code has been deposited in the SPP GitHub account at https://github.com/signalingpathways-project/ominer/.

\section{Mammalian Phenotype Ontology Analysis}

Genes mapping to MPO terms phenotypes were retrieved from MGI (Law et al., 2018). A hypergeometric test implemented in GraphPad Prism 7.0 was used to estimate the overrepresentation, relative to their distribution in all 691 nodes, of nodes encoded by MPO term-mapped genes among nodes with significant HCT intersections with aPU.1KO-induced or -repressed genes.

\section{Statistics}

The data are represented as the mean \pm standard deviation. For GTT and ITT assays, statistical significance was determined using repeated measures two-way ANOVA with Sidak correction for multiple comparison (GraphPad Prism v 9.3). $p<0.05$ was considered to be statistically significant. Pearson's correlation (PRISM software package v 7.0 (RRID: SCR_005375) was used to evaluate the correlation between differential expression values in the aPU.1KO $\mathrm{v}$ WT and those in the adipogenesis consensome.

\section{RESULTS}

\section{Generation of Adipocyte-Specific PU.1 Knockout Mice}

To investigate the systemic functions of aPU.1 in vivo, we generated mice with adipose-specific knockout of PU.1. Mice in which Spil gene exon 5 was flanked by loxP sequences were bred with mice carrying a transgene containing Cre recombinase under the control of adiponectin (Adipoq) gene promoter and enhancer (Figure 1A). Mice with adipose-specific knockout of PU.1 (adiponectinCre-PU. $1^{\mathrm{fl} / \mathrm{fl}}$ ) and littermate control mice $\left(P U .1^{\mathrm{fl} / \mathrm{fl}}\right.$ ) were used for the study. To confirm ablation of aPU.1 expression, we isolated the adipocyte fraction from gonadal adipose tissue. As shown in Figure 1B, PU.1 expression in the adipocytes of aPU.1KO mice was significantly down-regulated. We noticed that the PU.1KO adipocytes maintained a lower level of PU.1 expression. The Cre-LoxP mediated recombination might not be $100 \%$ efficient. Additionally, non-adipocytes with PU.1 expression, such as the macrophages, might contaminate the adipocyte fraction, likely in the form of lipid-laden foam cells or by sticking to adipocytes due to incomplete separation of cells to single cell suspension.

\section{Phenotype of Young Adult aPU.1KO Mice}

Young (4-5 months) aPU.1KO male (Figure 2A) or female (Supplementary Figure S1A) mice exhibited no difference in body weight, lean body mass or fat mass from floxed littermate controls. Moreover, glucose tolerance tests found no difference in glucose homeostasis (Figure 2B and Supplementary Figure S1B) between 4-5 months aPU.1 $\mathrm{KO}$ male or female mice and their floxed littermate controls. Using indirect calorimetry to measure energy expenditure however, we found that compared to wild-type littermates, male aPU.1KO mice had significantly higher average energy expenditure and basal energy expenditure under fasted and resting state (Figure 2C). This increase in energy expenditure was not contributed by increased physical activity, as there was no difference in ambulatory activity in these mice (Figure 2D). In female aPU.1KO mice, no difference of energy expenditure was observed (Supplementary Figure S1C).

\section{Deficiency of Adipocyte PU.1 Protects Against Age-Associated Obesity and Glucose Intolerance}

At 10-11 months of age, control male mice gained significantly more body weight than aPU.1KO mice (Figure 3A). Moreover, whereas lean body mass of aPU.1KO mice was indistinguishable from that of wild-type mice (Figure 3A), fat mass was significantly lower (Figure 3A), indicating that the difference of body weight was attributable primarily to a loss of adiposity. Fasted 10 months old aPU.1KO male mice exhibited significantly improved glucose tolerance compared with WT controls (Figure 3B) with significantly different area under the curve $(21,657 \pm 3,273$ vs. $15,609 \pm 2,697, p=0.006)$. Older aPU. $1 \mathrm{KO}$ mice also displayed higher insulin sensitivity (Figure $3 \mathrm{C}$ ) with 

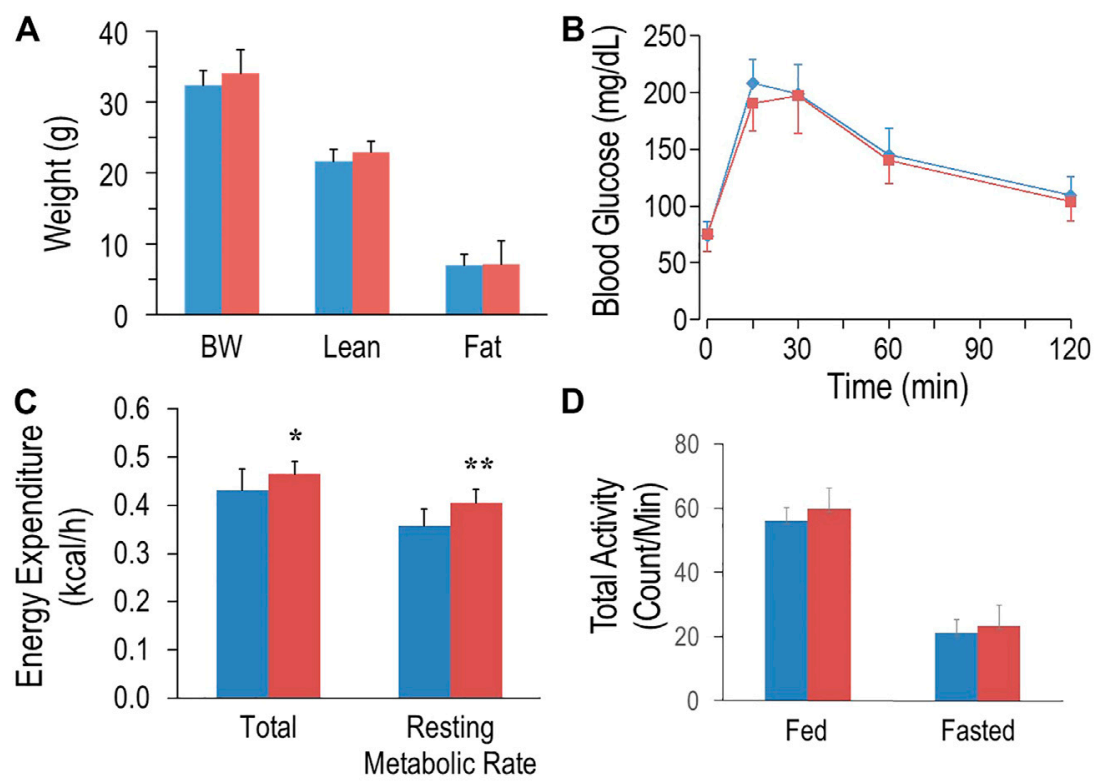

PU1 1 flif

AdipoqCre-PU1 $1^{\text {flfl }}$

FIGURE 2 | Adipocyte PU.1 Deficiency Has No Effect on Body Weight, Body Composition and Glucose Tolerance in Young Adult Male Mice. (A) Body weight (BW) and body composition of young adult (4-5 months of age) male AdipogCre-PU. $1^{1 / f / 1}$ mice $(N=8)$ and the control littermate PU. $1^{\mathrm{t} / \mathrm{fl}}$ mice $(N=8)$. (B) For glucose tolerance test, mice ( $N=8$ vs. 8) were fasted overnight and injected with glucose. Blood glucose was then measured. (C) Energy expenditure was determined using indirect calorimetry in the integrated Comprehensive Lab Animal Monitoring System (CLAMS; Columbus Instruments) in 4-5 months old male AdipoqCre-PU. $1^{\text {fltfl }}$ mice ( $N=8$ ) and the control littermate PU. $1^{\text {fltil }}$ mice $(N=8)$. (D) Total locomotor activity under fed or fasting condition. ${ }^{*} p<0.05,{ }^{* *} p<0.01$.

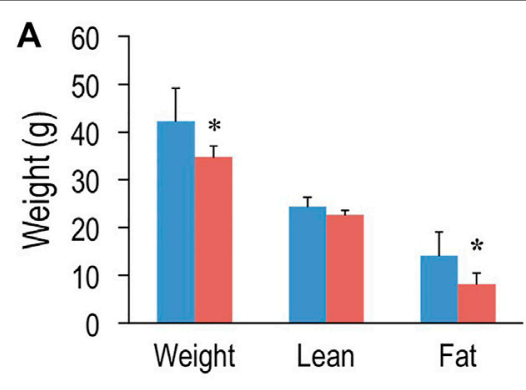

PU1/fill

AdipoqCre-PU1 ${ }^{\mathrm{fl} / \mathrm{fl}}$
B

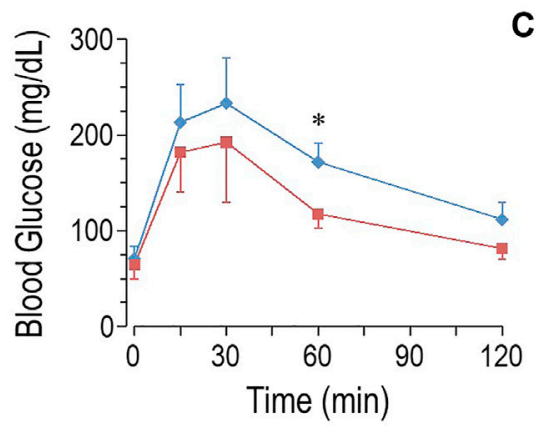

C

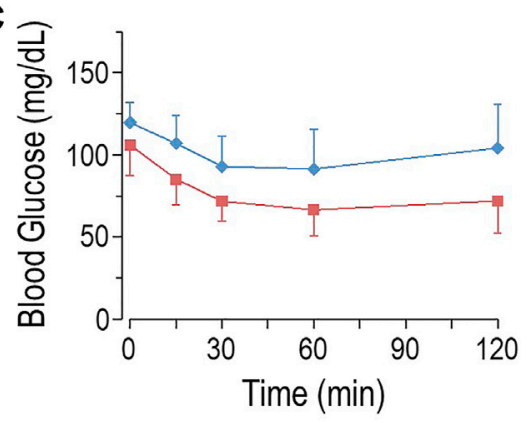

FIGURE 3 | Adipocyte PU.1 Deficiency Protects Mice Against Age-Associated Obesity and Insulin Resistance. (A) Body weight (BW) and body composition of 10 months old male AdipoqCre-PU. $1^{f / / f l}$ mice $(N=6)$ and the control littermate PU. $1^{f / f l}$ mice $(N=6)$. (B) For glucose tolerance test, 10 months old male mice $(N=7$ vs. 5$)$ were fasted overnight and injected with glucose ( $2 \mathrm{~g} / \mathrm{kg}$ body weight). Blood glucose was then measured. (C) For insulin tolerance test, 1 year old male mice ( $N=8$ vs. 7 ) were fasted for 4-hr and injected with insulin (1.0 IU/kg body weight). Blood glucose was measured afterwards. ${ }^{*} p<0.05$. 
A

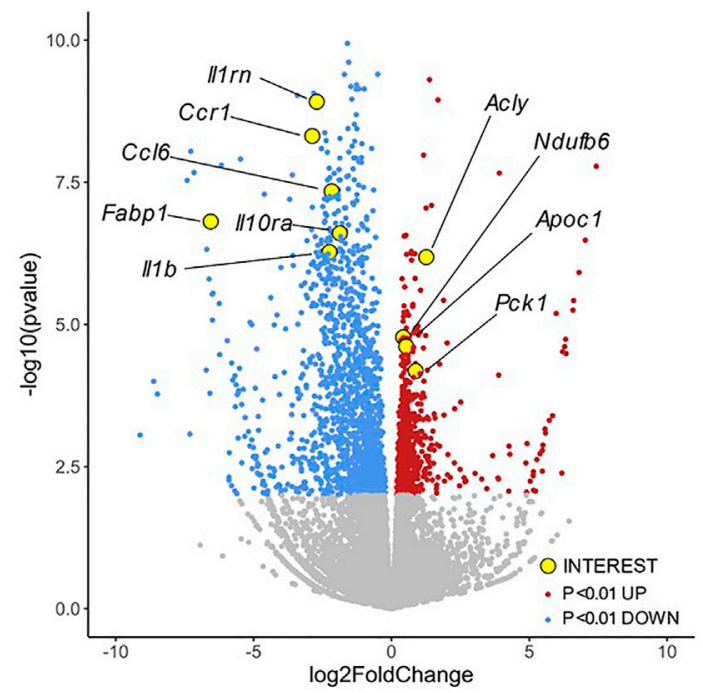

B

\begin{tabular}{lll}
\multicolumn{1}{c}{ Pathway Name } & pValue & FDR \\
Up-Regulated Genes & & \\
\cline { 1 - 2 } NR1H2 and NR1H3-mediated signaling & $1.44 \mathrm{E}-05$ & $4.91 \mathrm{E}-03$ \\
ChREBP activates metabolic gene expression & $1.73 \mathrm{E}-05$ & $4.91 \mathrm{E}-03$ \\
Glutathione conjugation & $1.41 \mathrm{E}-04$ & $2.59 \mathrm{E}-02$ \\
Activation of gene expression by SREBF (SREBP) & $1.84 \mathrm{E}-04$ & $2.59 \mathrm{E}-02$ \\
Transcriptional regulation of white adipocyte differentiation & $4.79 \mathrm{E}-04$ & $4.83 \mathrm{E}-02$ \\
PPARA activates gene expression & $7.03 \mathrm{E}-04$ & $4.83 \mathrm{E}-02$ \\
PKA-mediated phosphorylation of key metabolic factors & $6.11 \mathrm{E}-03$ & $2.87 \mathrm{E}-01$ \\
VEGF ligand-receptor interactions & $7.90 \mathrm{E}-03$ & $2.92 \mathrm{E}-01$ \\
Respiratory electron transport, ATP synthesis by chemiosmotic & $1.28 \mathrm{E}-02$ & $3.07 \mathrm{E}-01$ \\
coupling, and heat production by uncoupling proteins & & \\
& & \\
Down-Regulated Genes & $3.36 \mathrm{E}-11$ & $4.40 \mathrm{E}-08$ \\
\hline Extracellular matrix organization & $6.02 \mathrm{E}-10$ & $3.94 \mathrm{E}-07$ \\
Immune System & $1.31 \mathrm{E}-09$ & $5.72 \mathrm{E}-07$ \\
Neutrophil degranulation & $2.75 \mathrm{E}-09$ & $8.99 \mathrm{E}-07$ \\
Integrin cell surface interactions & $6.69 \mathrm{E}-09$ & $1.75 \mathrm{E}-06$ \\
Innate Immune System & $7.93 \mathrm{E}-07$ & $1.73 \mathrm{E}-04$ \\
Interleukin-10 signaling & $1.67 \mathrm{E}-06$ & $3.12 \mathrm{E}-04$ \\
Assembly of collagen fibrils and other multimeric structures & $2.61 \mathrm{E}-06$ & $4.26 \mathrm{E}-04$ \\
Platelet activation, signaling and aggregation & $2.11 \mathrm{E}-05$ & $2.40 \mathrm{E}-03$ \\
Interleukin-3, Interleukin-5 and GM-CSF signaling & $3.05 \mathrm{E}-05$ & $2.58 \mathrm{E}-03$ \\
Cytokine Signaling in Immune system & $6.73 \mathrm{E}-05$ & $4.38 \mathrm{E}-03$ \\
Regulation of Insulin-like Growth Factor (IGF) transport and uptake by & & \\
Insulin-like Growth Factor Binding Proteins (IGFBPs) & & \\
& &
\end{tabular}

FIGURE 4 | RNA-sequencing Analysis of Gene Expression Changes in the aPU.1KO Mice. (A) Volcano plot showing gene expression changes in adipocytes isolated from the gonadal adipose tissue, with key genes of interest highlighted in yellow. (B) Gene ontology analysis of differentially expressed genes $(p<0.01)$ showing highly enriched pathways. The false discovery rate (FDR) is used for multiple hypothesis testing, with a standard cutoff of FDR $<0.05$ for significant pathway enrichment.

significantly different area under the curve $(12,700 \pm 2,463$ vs. $9,677 \pm 2,023, p=0.03)$. Older female aPU.1KO mice were similar to WT controls with respect to body weight and fat mass (Supplementary Figure S2A). They did not present any improvement in glucose tolerance (Supplementary Figure S2B) or insulin sensitivity (Supplementary Figure S2C).

\section{Transcriptomic Analysis Identifies Regulation of Diverse Metabolic Transcriptional Programs by PU.1}

To investigate the metabolic phenotypes arising from loss of adipocyte PU.1, we performed RNA-sequencing of adipocytes isolated from epididymal adipose tissue of aPU.1KO and control male mice of more than 1 year of age. Significantly $(p<0.01)$ induced and repressed genes were visualized on a volcano plot (Figure 4A) and analyzed by Reactome Pathway Analysis (RPA; Figure 4B). RPA analysis of down-regulated genes displayed an enrichment of pathways involved in extracellular matrix and immune signaling, including interleukin-10 signaling (Figure 4B). Consistent with this, genes with well documented roles in these processes (Il1rn, Il10ra, Illb, Ccr 1, Ccl6) were highly repressed in aPU.1KO adipocytes (Figure 4A). In contrast, up-regulated genes were enriched for pathways involved in adipogenesis and lipogenesis, including Cebpa, Srebf1/SREBP, Nr1h3/LXRa, Mlxipl/ ChREBP, Acly, Apoc1 and Pck1 (Figures 4A,B). These results were consistent with our previous findings of the roles of PU.1 in driving expression of inflammatory genes and transcriptional suppression of adipogenesis in cultured adipocytes (Wang and Tong, 2008; Lin et al., 2012).

\section{Transcriptional Regulatory Network Analysis Illuminates Crosstalk of PU.1 With Adipogenic and Inflammatory Signaling Node Networks}

We next set out to identify direct PU.1 transcriptional targets within the RNA-Seq dataset, and to identify evidence for PU.1interacting proteins that coregulate expression of these transcriptional targets with PU.1. Signaling Pathways Project (SPP) consensomes are ranked consensus transcriptional signatures for signaling pathway nodes-receptors, enzymes, transcription factors and other nodes-computed from publicly archived omics datasets (Ochsner et al., 2019). As such, consensomes have value in identifying potential high confidence transcriptional targets (HCTs) for specific nodes or node families in a given biological system (Ochsner et al., 2020). To gain insight into members of the PU.1 transcriptional regulatory network, we next applied HCT intersection analysis to compute intersections between aPU.1KO-induced and aPU.1KO-repressed gene sets (FC $> \pm 1.5, p<0.05$ ) and a library of over 700 mouse HCT gene sets derived from archived transcriptomic or ChIP-Seq consensomes as previously described (Ochsner et al., 2019; Ochsner et al., 2020). We interpreted the size and significance of these intersections as evidence for loss or gain of function of a given signaling node or node family in aPU.1KO adipocytes and, by inference, a functional relationship with aPU.1.

Figure 5A shows a heatmap displaying selected intersections between the aPU.1KO up and down gene sets and SPP mouse node family transcriptomic (upper table panel) and node ChIPSeq (lower table panel) consensome HCTs. To assist in identifying candidate aPU.1-interacting nodes in WAT, 


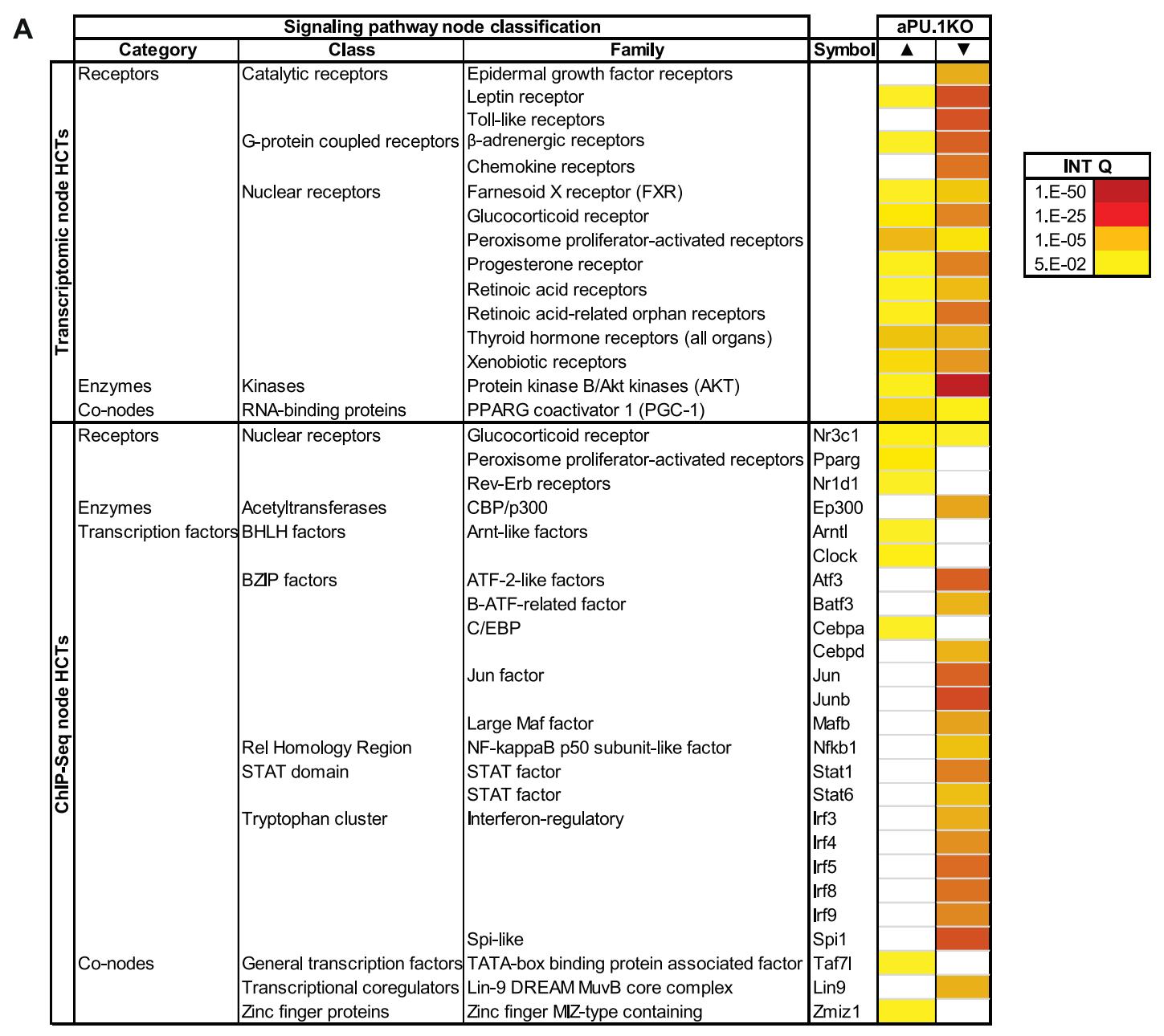

B

HCT intersection analysis of aPU.1KO-repressed genes

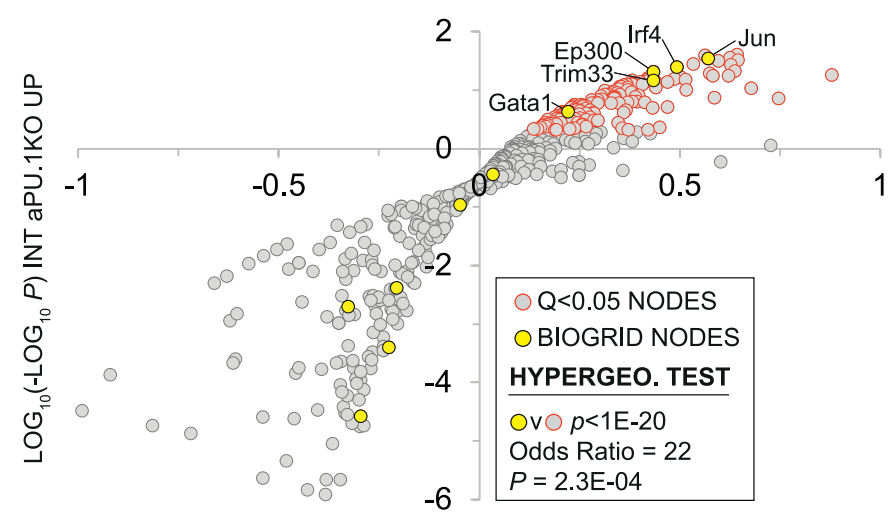

LOG OR INT APU.1KO UP

FIGURE 5 | High Confidence Transcriptional Target (HCT) Intersection Analysis Identifies PU.1-Modulated Metabolic and Inflammatory Transcriptional Regulatory Hubs. (A) HCT intersection Q-values (INT Q) for selected signaling pathway nodes or node families are indicated in the form of a heatmap. HCT intersection analysis was carried out as described in the Methods section. White cells represent $Q>5 E-2$ intersections. The intensity of the color scheme is proportional to the confidence of the intersection between high confidence transcriptional targets (HCTs) for a particular node and either (i) aPU.1KO-induced ( $\mathbf{\Delta}$ ) or (ii) aPU.1KO-repressed ( $\mathbf{v})$ gene sets. Lower confidence (smaller Q) intersections are towards the yellow end of the spectrum and higher confidence (larger Q) intersections are towards the red end of the spectrum. Full numerical data are in Supplementary Table S2. (B) Scatterplot showing enrichment of known BioGRID-curated PU.1 interacting nodes among nodes that have the most significant intersections with aPU.1KO-repressed genes. Refer to the text for details. 
Column P in Supplementary Table S1 represents percentiles of the mean WAT expression levels of each node derived from our RNA-Seq dataset. As an objective validation of our analysis, we benchmarked it against a list of 16 proteins identified by BioGRID (Oughtred et al., 2021) as mouse PU.1 interacting proteins (Supplementary Table S2, column Q). Figure 5B shows a regulatory footprint plot, in which signaling nodes that have significant HCT footprints with aPU.1KO-repressed genes are indicated in orange outline. In this plot, nodes that have the most highly enriched and significant regulatory footprints are located towards the top right of the plot. Reflecting the reliability of our predictions, the 16 BioGRID-sourced PU.1-interacting nodes (yellow data points in Figure $\mathbf{5 B}$ ) were enriched $(\mathrm{OR}=22$, $p=2.6 \mathrm{E}-04)$ among nodes with significant $(Q<1 \mathrm{E}-20)$ intersections with aPU.1-KO repressed genes (Figure 5B). In addition to the expected prominent footprint for PU.1 itself in the down-regulated gene set $(Q=1.9 \mathrm{E}-36)$, intersections with numerous canonical functional partners of PU.1 further validated our analysis. For example, robust intersections of aPU.1KO-repressed gene sets with transcriptomic HCTs for members of the toll-like (Wang et al., 2016), leptin (Dreyer et al., 2003), and chemokine (Ellis et al., 2010a) receptor and Protein kinase B/Akt (Rieske and Pongubala, 2001) families are consistent with previous studies implicating signaling through these nodes in PU.1 function (Figure 5A). Similarly, the increased energy expenditure of the aPU.1KO mice is reflected in the strong footprint within the aPU.1KO-induced gene set of transcriptomic HCTs for members of the PPARG coactivator 1 (PGC-1) family, which are well known mediators of metabolic control (Lin et al., 2005). Moreover, reflecting adrenergic stimulation of thermogenesis (Collins and Surwit, 2001) as well as repression of inflammatory cytokines (Ağaç et al., 2018), we observed $\beta$-adrenergic footprints in both aPU.1KOinduced $(Q=1 \mathrm{E}-4)$ and - repressed $(Q=7 \mathrm{E}-33)$ gene sets (Figure 5A).

Similarly, in the ChIP-Seq HCTs, robust intersections of aPU.1KO-regulated genes with HCTs for members of the interferon regulatory factor (IRF) (Marecki and Fenton, 2000), STAT (Nguyen and Benveniste, 2000), AP-1 (Steidl et al., 2006), Atf (Labzin et al., 2015), C/EBP and GATA (Wang and Tong, 2008) transcription factor families are consistent with canonical PU.1 biology. Given our previous report that PU.1 functions synergistically with GATA transcription factors to inhibit adipogenesis (Wang and Tong, 2008), we were also interested to note intersections of aPU.1KO-regulated genes with HCTs for GATA family members (Figure 5B). Congruent with our metabolic studies of aPU.1KO mice (Figures 2, 3), aPU.1KO-induced genes contained footprints for several nodes with familiar roles in the context of whole body energy metabolism, including Pparg (3E-7), Nr3c1/GR (1.2E-05) and Cebpa (1.2E-5). Strikingly, four nodes with known roles in circadian rhythms (Clock (Debruyne, 2008), 1.2E-5; Nr3c1/GR (So et al., 2009), 1.2E-05; Nr1d1/REVERB (Ramakrishnan and Muscat, 2006), 1.1E-04 and Arntl/ BMAL1 (Menet et al., 2014), 2.1E-04) also had appreciable intersections with the aPU.1KO-induced genes (Figure 5A and Supplementary Table S2).
In addition to corroborating canonical PU.1 biology, our analysis suggested the possibility of crosstalk of PU.1 with signaling nodes with which it has no previously established functional relationships. For aPU.1KO-induced genes these included transcriptomic HCT intersections with the PGC-1 family and ChIP-Seq HCT intersections for Zbtb11, Taf3 and Zfp57. Similarly, for aPU.1KO-repressed genes, we noted intersections with HCTs for members of the E2A-related factor family and the MuvB complex members Lin9 and E2f4 (Supplementary Table S2).

Some of the intersections may be attributable to transcriptional induction or repression of their encoding genes in the absence of PU.1. For example, the footprint for Cebpa in the PU.1-induced genes may be explained in part by the fact that its gene was upregulated in the knockout cells (Cebpa aPU.1KO v WT $\log 2$ FC $=0.35$; Supplementary Table S1). Similarly, the footprints for Irf5, Irf8, Mafb and Mef2c in the aPU.1-repressed genes may reflect downregulation of the genes encoding these nodes in the knockout cells (aPU.1KO v WT log2 FCs: Irf5, -2.6; $\operatorname{Irf8,}-0.83 ;$ Mafb, -1.2 ; Mef2c, -0.95 ; Supplementary Table S1). The vast majority of nodes that had significant HCT intersections with aPU.1KO-regulated genes were however not transcriptionally regulated, suggesting that a post-translational mechanism contributes to their loss of function in the absence of PU.1. Collectively these data indicate repressive, protein-level cross-talk of PU.1 with adipogenic/lipogenic signaling nodes on the one hand, and on the other, positive cross-talk with distinct classes and families of inflammatory signaling nodes.

\section{Consensome Analysis Identifies Broad Scale Direct Regulation by PU.1 of Adipogenic Gene Expression}

We previously showed that PU.1 inhibits adipogenesis (Wang and Tong, 2008). With that in mind, our RNA-Seq analysis highlighted numerous aPU.1 KO-regulated genes that represented potentially novel, previously uncharacterized modulators of adipogenesis. We next wished to adopt a reduced-bias approach to explore this possibility in more detail. Using our previously-described consensome algorithm (Ochsner et al., 2019), we used five archived datasets (GSE2192, GSE60745, GSE14004, GSE12929, GSE20696) to generate a 3T3-L1 adipogenesis consensome, which ranks $\sim 12,500$ genes according to the frequency with which they are upregulated or downregulated across independent transcriptomic 3T3-L1 adipogenic datasets (Supplementary Table S3 contains 3T3-L1 adipogenesis consensome genes with $p<0.05, n=9,152$ ). Within the 3T3-adipogenesis consensome we designated $\mathrm{Q}<0.05$ genes with a mean FC $>2(\log \mathrm{FC}>1)$ as 3T3-L1 adipogenesis induced confidence transcripts (3T3-ADIPICTs, $n=508$; Supplementary Table S3, column I) and Q $<0.05$ genes with a mean $\mathrm{FC}<0.5(\log \mathrm{FC}<-1)$ as adipogenesis repressed confidence transcripts (3T3-ADIPRCTs, $n=100$; Supplementary Table S3, column J). We first benchmarked the 3T3-L1 adipogenesis consensome against a set of 200 genes designated as hallmark adipogenesis-induced transcripts by the GSEA (Subramanian et al., 2005) resource (GSEA 

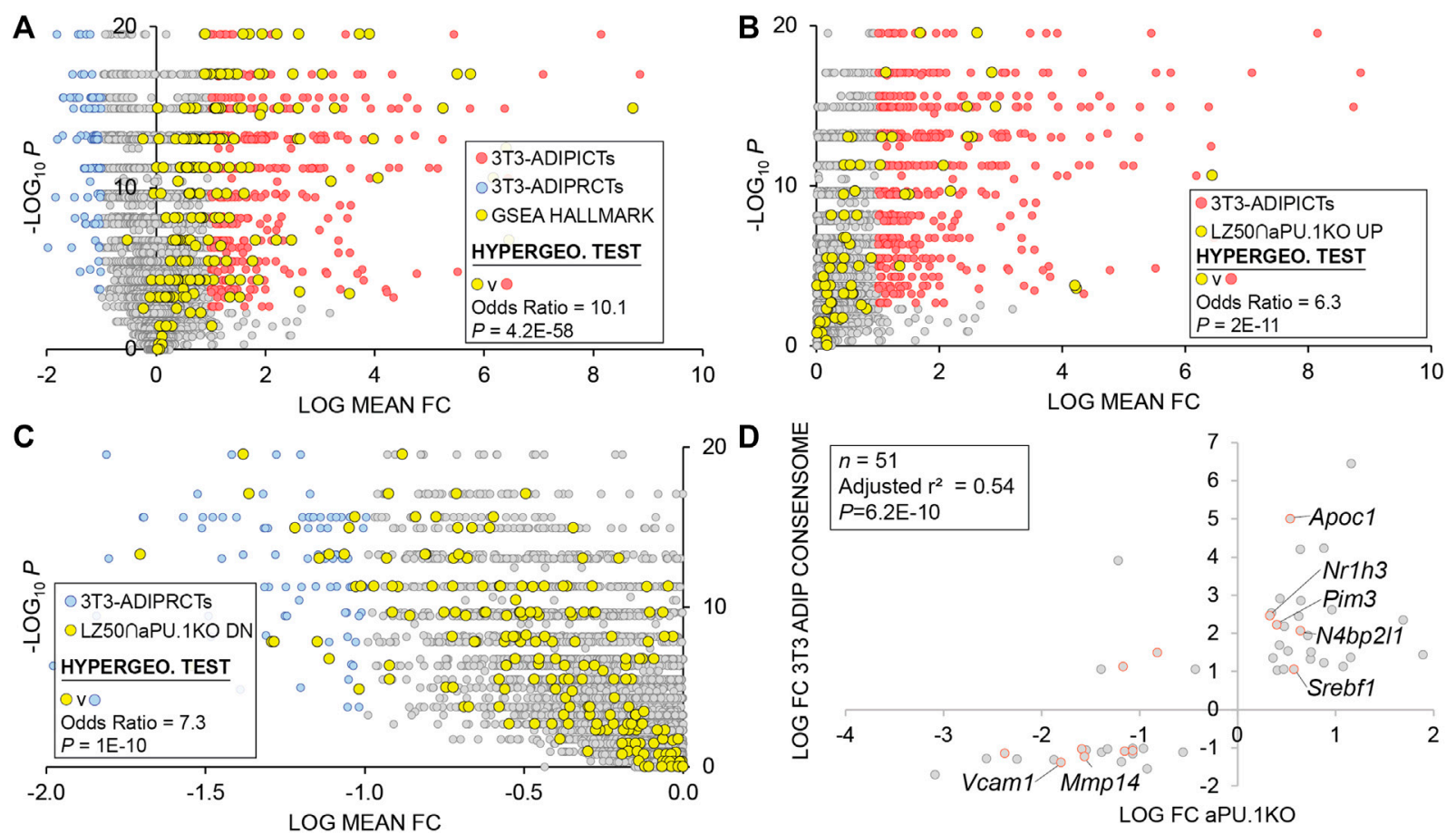

FIGURE 6 | 3T3-L1 Adipogenic Differentiation Consensome. The mouse 3T3-L1 adipogenesis transcriptomic consensome ranks mouse genes based on their discovery rates across five independent, publicly archived 3T3-L1 adipogenesis transcriptomic datasets. Hypergeometric test odds ratio and associated $p$-value are indicated in each panel. (A) Validation of the 3T3-L1 adipogenesis consensome against the GSEA adipogenesis Hallmark gene set. (B) Over-representation of aPU.1KOinduced LZ50 genes among 3T3-ADIPICTs. (C) Over-representation of aPU.1KO-repressed LZ50 genes among 3T3-ADIPRCTs. (D) Correlation between aPU.1KO log FC and adipogenesis log mean FC.

TABLE 1 | Selected PU.1 regulated genes with elevated rankings in the 3T3-L1 adipogenesis transcriptomic consensome.

\begin{tabular}{|c|c|c|c|c|c|c|c|}
\hline \multicolumn{4}{|c|}{ Target } & \multirow{2}{*}{$\frac{\mathrm{aPU} .1 \mathrm{KO}}{\mathrm{LFC}}$} & \multicolumn{3}{|c|}{ 3T3-ADIP CONSENSOME } \\
\hline Category & Class & Family & Symbol & & LMFC & $\%$ ile & $p$ \\
\hline \multicolumn{8}{|c|}{ aPU1.KO-induced } \\
\hline \multirow[t]{3}{*}{ Enzymes } & Dehydrogenases & 3 beta hydroxysteroid dehydrogenases & Hsd3b7 & 0.37 & 0.57 & 94 & $9.64 \mathrm{E}-14$ \\
\hline & Reductases & Glyoxylate and hydroxypyruvate reductases & Grhpr & 0.40 & 1.04 & 92 & 5.53E-12 \\
\hline & Regulatory factors & NEDD4 binding proteins like & N4bp2/1 & 0.64 & 2.07 & 90 & 5.87E-12 \\
\hline \multirow[t]{4}{*}{ Co-nodes } & Developmental proteins & Testis development related protein & $T d r p$ & 0.45 & 0.89 & 87 & $2.32 \mathrm{E}-10$ \\
\hline & Glycoproteins & Glycoprotein integral membrane & Ginm1 & 0.45 & 1.27 & 99 & 8.73E-18 \\
\hline & Membrane proteins & Tetraspanin & Tspan12 & 0.48 & 2.18 & 87 & $2.32 \mathrm{E}-10$ \\
\hline & Stress response factors & DnaJ heat shock protein (Hsp40) member & Dnaja3 & 0.40 & 0.67 & 99 & 8.73E-18 \\
\hline \multicolumn{8}{|c|}{ aPU1.KO-repressed } \\
\hline \multirow[t]{2}{*}{ Receptors } & G protein coupled receptors & G protein-coupled receptor & Gpr176 & -3.09 & -1.71 & 95 & 5.59E-14 \\
\hline & Ligands & FAT atypical cadherin & Fat4 & -0.92 & -1.54 & 74 & 4.95E-07 \\
\hline \multirow[t]{3}{*}{ Enzymes } & ADP ribosyltransferases & Poly [ADP-ribose] polymerases (PARP) & Parp14 & -0.56 & -1.11 & 95 & 5.59E-14 \\
\hline & Dehydratases & 3-hydroxyacyl-CoA dehydratases & Hacd4 & -1.07 & -1.15 & 80 & 1.61E-08 \\
\hline & GTPases & RAB, member RAS oncogene & $R a b 7 b$ & -2.56 & -1.28 & 80 & 1.61E-08 \\
\hline \multirow[t]{6}{*}{ Co-nodes } & $\begin{array}{l}\text { Apoptosis and apoptosis } \\
\text { regulators }\end{array}$ & Niban apoptosis regulator & Niban1 & -1.13 & -1.01 & 90 & 5.87E-12 \\
\hline & $\begin{array}{l}\text { Cytoskeleton components and } \\
\text { regulators }\end{array}$ & FERM domain containing & Frmd4a & -1.07 & -1.06 & 95 & 5.59E-14 \\
\hline & Membrane proteins & CKLF like MARVEL transmembrane domain containing & Cmtm3 & -1.55 & -1.05 & 97 & 1.17E-15 \\
\hline & Pleckstrin domain & Pleckstrin homology domain containing & Plekho2 & -1.59 & -1.03 & 98 & 2.44E-16 \\
\hline & Other co-nodes & MAM domain containing & Mamdc2 & -2.25 & -1.29 & 80 & 1.61E-08 \\
\hline & & Vesicle amine transport & Vat1 & -0.86 & -1.11 & 80 & 1.61E-08 \\
\hline
\end{tabular}


HALLMARK; Supplementary Table S3, column Q). Validating the 3T3-L1 adipogenesis consensome, the GSEA HALLMARK gene set was robustly over-represented among 3T3-ADIPICTs (OR = 10.1, $p=4.2 \mathrm{E}-58$; Figure 6A). Many of the aPU.1KO-regulated 3T3-ADIPICTs are immediately familiar in the context of adipogenesis, including Cidec (Keller et al., 2008), Adipoq (Hu et al., 1996) and Acsl1 (Ellis et al., 2010b). Interestingly, numerous aPU.1KOinduced (Supplementary Table S3, column R) and aPU.1KO-repressed (Supplementary Table S3, column S) genes have 3T3-L1 adipogenesis consensome rankings that are comparable to or exceed those of classic adipogenic markers, but have potential roles in adipogenesis that to date are unexplored in the research literature (Table 1).

To focus on direct aPU.1 targets among genes with elevated rankings in the 3T3-adipogenesis consensome, we next percentilized peak call heights $(n=679)$ from a previously published PU.1 3T3-L1 ChIP-Seq analysis by Lazar and colleagues (DiSpirito et al., 2013) and mapped these to genes in the 3T3-L1 adipogenesis consensome (Supplementary Table S3, column T). For the purposes of subsequent statistical analyses, the 50th percentile of this gene set $(n=444)$ is referred to here as LZ50. Consistent with broad, direct antagonism by PU.1 of the 3T3-L1 adipogenic transcriptional program, we observed robust enrichment of aPU.1KO-induced LZ50 genes $(n=78)$ in 3T3ADIPICTs (Figure 6B; OR $=6.3, p=2.2 \mathrm{E}-11$ ) and of aPU.1KOrepressed LZ50 genes $(n=293)$ among 3T3-ADIPRCTs (Figure 6C; $\mathrm{OR}=7.3, P=1 \mathrm{E}-10$ ). Further reflecting the role of aPU.1 as an important direct regulator of adipogenic differentiation, we observed a clear positive correlation (adjusted $r^{2}=0.54, P=6 \mathrm{E}-10$, Pearson's correlation) between aPU.1KO log FCs and mean 3T3-adipogenesis consensome log FCs for 3T3-ADIPRCTs or 3T3-ADIPICTs in the 50th percentile of PU.1 3T3-L1 peaks ( $n=51$. Figure 6D). Studying these 51 genes further, we identified two prominently pro-adipogenic members of the 3T3-ADIPICTs subset, Srebf1 (encoding SREBP1) and Nr1h3 (encoding LXRa), that have not been previously appreciated as direct PU.1 transcriptional targets. Similarly, uncharacterized candidate direct PU.1 targets within the 3T3-ADIPRCT gene subset included Vcam1, previously shown to mediate adhesion of inflammatory macrophages to adipocytes as a potential mechanism driving insulin resistance (Chung et al., 2017), and Mmp14, whose role in adipogenic collagen turnover has been linked to obesity (Chun et al., 2010). Collectively, our in vivo analysis confirms and adds value to previous in vitro studies implicating PU.1 as an antiadipogenic, pro-inflammatory driver of gene expression in adipocytes.

\section{SPP Web Resource Facilitates the Generation of Novel Hypotheses Around PU.1 Regulation of Adipogenic Gene Expression}

To make full use of the adipogenesis consensome for hypothesis generation around aPU.1 regulation of adipogenic gene expression, it is important that the data points be placed in the context of data points from other mouse adipose transcriptomic experiments. For each gene in Supplementary Table S3 therefore, column U links to an SPP website interface showing the specific experimental data points for that gene from the 3T3-L1 adipogenesis expression profiling datasets. In addition, the interface includes data points from transcriptomic experiments in mouse adipose tissue or cell lines involving genetic or small molecule perturbation of various receptor and enzyme signaling nodes, as well as data points from metabolic challenges such as cold exposure. For insight into nodes directly regulating expression of genes in the adipogenesis consensome, Supplementary Table S3 column V links to an interface showing data points from ChIP-Seq experiments carried out in mouse adipose tissue or cell lines. Data points in both the transcriptomic and ChIP-Seq interfaces link to contextual pop-up windows, which in turn point to the full source datasets on the SPP website.

\section{Confirmation of PU.1 Regulation of Pro-inflammatory Cytokines and} Thermogenesis Genes in Adipose Tissues

Given the global transcriptional repression of cytokine production in aPU.1KO adipocytes indicated by the RNA-Seq analysis (Figure 4), we next used quantitative real-time RTPCR (Q-PCR) to validate this observation. We confirmed the reduction of the expression of $C c l 2 / \mathrm{MCP}-1$ and $T n f$ in adipocytes isolated from epididymal WAT of 1 yo male aPU.1KO mice (Figure 7A). Since aPU.1KO mice exhibited elevated energy expenditure, we speculated that we might also observe induction of thermogenic expression programs in brown adipose tissue (BAT) of aPU.1KO mice. Consistent with this hypothesis, Q-PCR analysis identified transcriptional induction in aPU.1KO BAT of two key thermogenic genes, Ppargcla/PGC-1a and Ucp1 (Figure 7B). These results suggest a molecular mechanism underlying PU.1 regulation of adipocyte inflammation and thermogenesis and offer an explanation of the phenotype of the aPU.1KO mice.

\section{Integrated Analysis of aPU.1KO Transcriptional Regulatory Networks and Mouse Phenotypes}

The Mammalian Phenotype Ontology (MPO) (Smith and Eppig, 2009), uses evidence from the research literature to assign specific metabolic and physiological functions to the products of mouse genes. As such, MPO annotations represent a potentially powerful approach to inferring the metabolic impact of transcriptional regulatory networks in the aPU.1KO mouse. Given the increased energy expenditure of aPU.1KO mice (Figure 2), we first wished to gather evidence for nodes that are transcriptional mediators of increased whole body energy expenditure in these mice. To do this we examined the intersection between nodes that had significant intersections with aPU.1KO-UP genes, and those encoded by genes whose 


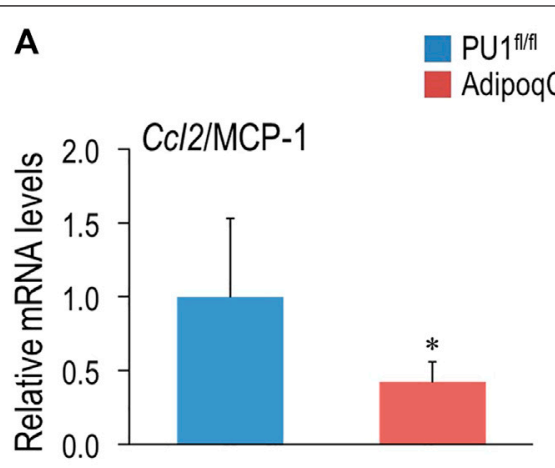

PU1 1/fil

AdipoqCre-PU1 $1^{\text {fl/fl }}$
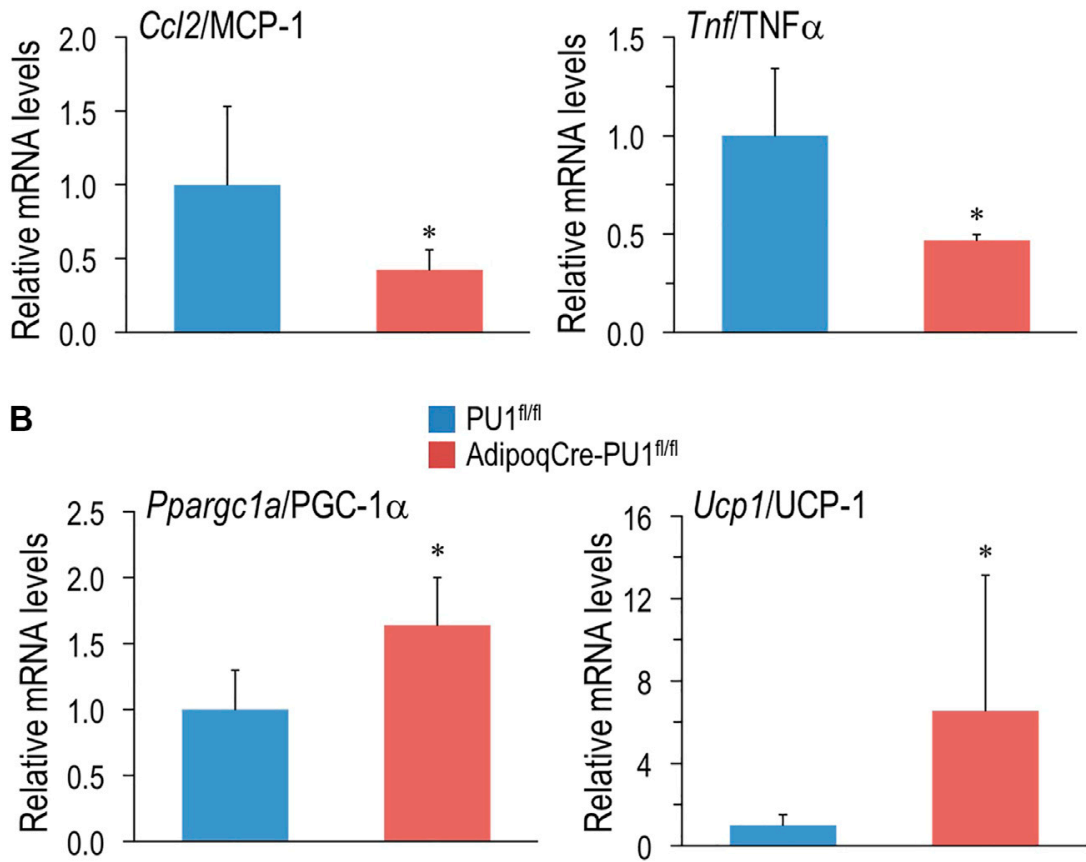

FIGURE 7 | Loss of PU.1 in Adipocytes Reduces Inflammatory Gene Expression and Promotes Thermogenic Gene Expression. (A) Adipocytes were isolated from the gonadal adipose tissue and mRNA expression of ccl2/MCP-1 and TNFa were measured using real-time RT-PCR. (B) Brown adipose tissue mRNA expression of PGC- $1 \alpha$ and UCP1 and TNF $\alpha$ were measured using real-time RT-PCR. $N=5$ vs. $5,{ }^{*} p<0.05$.

disruption in mice mapped to the MPO term "abnormal energy expenditure" (AEE; Supplementary Table S2, column R). Consistent with the increased energy expenditure in the aPU.1KO mice, nodes encoded by AEE-mapped genes were strongly enriched among the top ranked nodes that had significant intersections with aPU.1KO-induced genes $(\mathrm{OR}=$ 14, $\mathrm{P}=9 \mathrm{E}-5$, Figure 8A) but not the aPU.1KO-repressed genes. These nodes included the nuclear receptors Pparg (Kubota et al., 1999) and Ppara (Finck et al., 2005), the circadian clock regulators Clock (Turek et al., 2005) and Arntl (Shimba et al., 2011), and the C/EBP family member Cebpa (Wang et al., 1995) which, as previously noted, was strongly transcriptionally induced in adipocytes in the aPU.1KO mice (Figure 2).

Next, to identify potential transcriptional mediators of thermogenic gene expression in the aPU.1KO mice (Figure 4), we compared nodes that had significant intersections in aPU.1KO-UP genes with those encoded by a set of genes that mapped to at least one of the MPO terms "decreased brown adipose tissue amount", "decreased core body temperature" or "impaired adaptive thermogenesis" (collectively referred to as THERMO genes; Supplementary Table S2, column S). Consistent with the induction of BAT thermogenic genes in the aPU.1KO animals, nodes encoded by THERMO genes were strongly enriched among the top ranked nodes that had significant intersections with aPU.1KO-induced genes $(\mathrm{OR}=20, P=2 \mathrm{E}-05$; Figure $8 \mathrm{~B})$ but not aPU.1KO- repressed genes. These included the nuclear receptor Pparg (Imai et al., 2004), the C/EBP family transcription factor Cebpa (Wang et al., 1995), the general transcription factor Taf7l (Zhou et al., 2014) and the cohesin complex member Stag1 (Remeseiro et al., 2012). Collectively these data indicate that a non-redundant role for PU.1 in repressing a transcriptional regulatory network driving thermogenic gene expression in vivo.

Node HCT intersection analysis had previously indicated that numerous inflammatory transcription factors with roles in cytokine production were functionally impacted by the loss of PU.1 (Figure 5A). To gain insight into the PU.1 adipocyte transcriptional hub supporting inflammatory cytokine production in vivo, we compared nodes that had significant intersections with aPU.1KO-DOWN genes and those encoded by genes whose disruption in mice mapped to the MPO term "abnormal cytokine levels" (ACL; Supplementary Table S2, column T). Reflecting transcriptional repression of numerous inflammatory cytokine genes in the aPU.1KO mice (Figures 4, 7A, and Supplementary Table S1), nodes encoded by ACL genes were strongly enriched among the top ranked nodes that had significant intersections with aPU.1KO-repressed genes $(\mathrm{OR}=$ $11, P=3 \mathrm{E}-05$; Figure 8C) but not the aPU.1KO-induced genes. These included members of the IRF (Irf3, Irf5), STAT (Stat1, Stat6) and AP-1 (Jun, Junb) transcription factor families, as well as Fosl1, Cebpd and Nfkb1. Collectively these data indicate that PU.1 has a non-redundant role in anchoring a transcriptional 

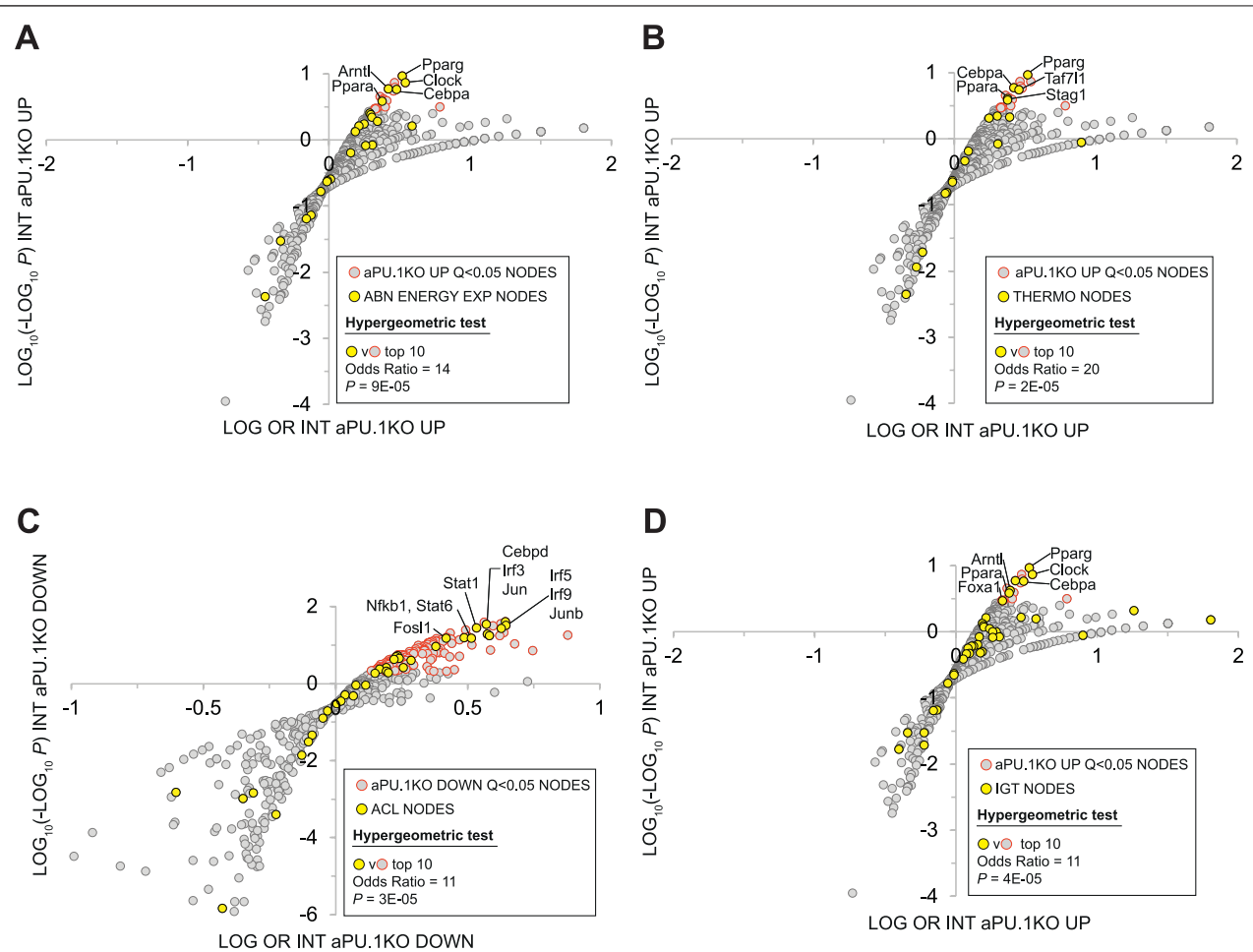

FIGURE 8 | Mammalian Phenotype Ontology Analysis Of Nodes With Significant HCT Intersections With aPU.1KO-Induced and Repressed Gene Sets. (A) Enrichment of nodes encoded by genes that map to the MPO term "abnormal energy expenditure" (AEE) among nodes that have significant HCT intersections with aPU.1KO-induced genes. (B) Enrichment of nodes encoded by genes that map to the MPO terms "decreased brown adipose tissue amount", "decreased core body temperature" or "impaired adaptive thermogenesis" (collectively referred to as THERMO genes) among nodes that have significant HCT intersections with aPU.1KO-induced genes. (C) Enrichment of nodes encoded by genes that map to the MPO term "abnormal cytokine levels" (ACL) among nodes that have significant HCT intersections with aPU.1KO-repressed genes. (D) Enrichment of nodes encoded by genes that map to the MPO term "impaired glucose tolerance" (IGT) among nodes that have significant HCT intersections with aPU.1KO-induced genes. Please refer to the Methods for details of the analysis.

regulatory network supporting adipocyte cytokine gene expression in vivo.

We showed that aPU.1KO mice exhibited improved glucose homeostasis (Figure 3). To gather evidence for the specific signaling nodes contributing to this phenotype, we compared nodes that had significant intersections in aPU.1KO-UP genes with those encoded by genes mapped to the MPO term "impaired glucose tolerance" (IGT; Supplementary Table S2, column U). Consistent with improved glucose homeostasis in the aPU.1KO mice, nodes encoded by IGT genes were strongly over-represented among nodes with $\mathrm{Q}<0.01$ intersections with aPU.1KO-induced genes $(\mathrm{OR}=6, p=5.7 \mathrm{E}-05$, Figure 8D). Confirming the link between improved glucose tolerance and energy expenditure, these included the five previously identified AEE nodes (Pparg, Ppara, Clock, Arntl and Cebpa), in addition to Foxal.

\section{DISCUSSION}

In this study, we set out to investigate the role of the transcription factor PU.1 in adipocytes in vivo, particularly during aging. We observed that although young (4-5 months) aPU.1KO mice had no overt phenotypes with respect to body weight, body composition, or glucose homeostasis, they did exhibit elevated energy expenditure. Moreover, at around 1 year of age, aPU.1KO mice were protected against age-associated obesity, adipose tissue inflammation, and insulin resistance. Mechanistically, and consistent with their elevated energy expenditure, we found that loss of adipocyte PU.1 suppressed inflammatory transcriptional programs in WAT and promoted thermogenic gene expression in BAT. Using a combination of conventional, literature-based pathway analysis and a novel 'omics datasetcentric analytic platform, we identified numerous PU.1modulated signaling systems and downstream functional pathways that shed mechanistic light on these phenotypes.

Consistent with the enhanced energy expenditure of aPU.1KO mice, we identified a robust transcriptional footprint within aPU.1KO-induced genes for members of the PGC-1 family (Figure 5A), which are well known transcriptional drivers of thermogenesis and energy metabolism (Puigserver et al., 1998). This represented strong evidence, validated by subsequent Q-PCR analysis (Figure 7A), that PU.1 suppresses thermogenic transcriptional programs in mice. To afford insight into the functional pathways involved, Supplementary Table S2 column L indicates aPU.1KO-induced genes that are HCTs for the PGC-1 family. Many of these are familiar players in 
cellular energy metabolism that have emerged from studies in the research literature. The induction of $N d u f b 6, N d u f b 10$ and $N d u f s 6$, for example, reflects our finding from RPA analysis that respiratory electron transport chain pathway genes were enriched among aPU.1KO-induced genes (Figure 4A). Moreover, upregulation of the transferrin (Trf) gene may reflect potential endocrine or paracrine signaling from the white adipocytes to activate brown or beige adipocyte thermogenesis in aPU.1KO mice (Qiu et al., 2020). The power of consensome analysis, however, is that it illuminates genes for which a specific function is not described in the research literature, but for which, based upon close regulatory relationship with a node family computed from 'omics datasets, that function can be inferred with a high level of confidence. This is the case with genes such as Blcap, Gkap1, Procal and others, for which, as aPU.1KO-induced PGC-1 HCTs, a functional contribution to enhanced bioenergetics of the aPU.1KO animals can be reasonably surmised. Supporting this assertion, and confirming the clinical relevance of our study, the human ortholog of Fam13a (aPU.1KO $\operatorname{logFC}=1.3$; PGC-1 family consensome 95th percentile) has been recently shown to regulate fat distribution and metabolic traits through its action on adipose tissue (Fathzadeh et al., 2020). In summary, we conclude that activation of PGC-1 family signaling in aPU.1KO mice contributes to the increased energy expenditure, reduced adiposity, improved glucose homeostasis and insulin sensitivity of aPU.1KO mice, and ultimately protects against age-related metabolic abnormalities in these animals.

Transcriptomic analysis has cast PU.1 in the global maintenance of pro-inflammatory transcriptional programs in response to sepsis or lipopolysaccharide stimulation (Karpurapu et al., 2011). Although transcriptional induction by PU.1 of proinflammatory factors, including Tnf, Illb and $I l 6$, as well as Ccl3 and $\mathrm{Ccl} 2$ has been extensively documented in macrophages and other immune cell lineages (Karpurapu et al., 2011), there is increasing interest in its role as a pro-inflammatory transcription factor in adipose tissue. Building on our previous study showing that PU.1 activates inflammatory cytokine expression in cultured adipocytes (Lin et al., 2012), our current study shows that depletion of adipocyte PU.1 results in broad, transcriptomescale suppression of inflammatory programs in adipocytes. Since inflammation is a key mediator for insulin resistance and metabolic syndrome (Hotamisligil, 2017), suppression of this inflammatory transcriptional program likely contributes to the phenotypes of the aPU.1KO mice. Going to the underlying mechanism, we found that the expression levels of many proinflammatory cytokines driven by the PU.1 transcription factor, such as illb, tnf and $c c l 2 / \mathrm{MCP}-1$, are down regulated in the adipocytes of aPU.1KO mice (Figures 4, 7). IL-10 signaling, which has been shown to inhibit thermogenesis and energy expenditure in adipocytes (Rajbhandari et al., 2018), was also downregulated in PU.1-deficient adipocytes (Figure 4B). Members of the NOD-like family of receptors have prominent roles in the transcriptional regulation of inflammasome pathways. Our transcriptomic analysis identified significant down-regulation of genes encoding two members of the NODlike family, Ciita and Nrpl3, in aPU.1KO adipocytes
(Supplementary Table S1). Similarly, consistent with the strong TLR regulatory footprint in the aPU.1KO-repressed genes (Figure 5A), genes encoding four members of the TLR family (Tlr1, Tlr6, Tlr7 and Tlr8) are repressed in the aPU.1KO. Given that TLRs (Kim et al., 2012), Ciita (Deng et al., 2013) and Nrpl3 (Stienstra et al., 2010) are known to be induced in obese adipose tissue or to support adipocyte inflammation, it can be justifiably speculated that their transcriptional induction in adipocytes makes an important contribution to PU.1's action in promoting inflammation and insulin resistance.

On a broader scale, HCT intersection analysis (Figure 5A), validated by integration with literature-based mouse phenotype annotations (Figure 8C), reflects the profound impact of loss of PU.1 on the function of numerous inflammatory node families. For example, aPU.1KO-downregulated genes contain a sizeable regulatory footprint for members of the IRF transcription factor family (Figure 5A and Supplementary Table S2). Given that the roles of members of the IRF family in the regulation of adipogenesis, inflammation and thermogenesis in adipocytes are well-documented (Eguchi et al., 2008; Kumari et al., 2016), we interpret the presence of this footprint as evidence for strong, network-level interactions between PU.1 and IRFs in adipocytes. On the other hand, transcription factors that suppress inflammatory gene expression, such as PPAR $\gamma$ (Lefterova et al., 2010) and LXRs (Heinz et al., 2010), have DNA binding sites adjacent to PU.1 binding sites, potentially reflecting mutual functional antagonism. Given that we observed evidence for activation of both PPAR and LXR in response to aPU.1 loss of function (Figure 4B), we speculate that aPU.1 may also drive inflammation through the suppression of PPAR and LXR transcription factors

Although 'omics datasets have intrinsic value for metabolic research, they realize their full value when integrated with existing data resources to facilitate the generation of hypotheses around metabolic signaling pathways not explored in the research literature. A unique aspect of our RNA-Seq dataset is that rather than limiting it to a standalone analysis of aPU.1KOregulated genes, we have placed it in the context of millions of regulatory data points curated from archived 'omics datasets by the SPP cell signaling knowledgebase (Love et al., 2014). Annotation of aPU.1KO-regulated gene list (Supplementary Table S1) according to the SPP classification, for example, provides for an immediate appreciation of the diversity of cellular functions impacted by PU.1 depletion. Similarly, HCT intersection analysis (Figure 5 and Supplementary Table S2) affords a unique perspective on the various receptors, enzymes, transcription factors and co-nodes that are functionally impacted by PU.1 depletion and which, by extension, are candidate PU.1-interacting proteins. Finally, the adipose-centric SPP transcriptomic and ChIP-Seq Regulation Reports to which the 3T3-L1 adipogenic consensome (Supplementary Table S3) links provide the user with a rich, contextual perspective to generate hypotheses around transcriptional regulation of novel effectors of adipose tissue biology. By integrating these three data resources in a single study, we provide for a unique perspective on PU.1-dependent transcriptional 
regulatory networks in adipocytes, and an appreciation of how diverse signaling nodes impact expression of a specific PU.1 target gene (Ochsner et al., 2019).

The collective value of our supplementary material to researchers in generating novel metabolic hypotheses can be illustrated with reference to Gpr176, identified in Supplementary Table $\mathbf{S 1}$ as a gene encoding a member of the $G$ protein-coupled receptor family that is strongly transcriptionally dependent upon PU.1. With the exception of a role in the regulation of circadian clock in the suprachiasmatic nucleus (Doi et al., 2016), the function of this receptor is largely uncharacterized. The 3T3-L1 adipogenesis consensome ranks Gpr176 445th of 12525 genes (mean log FC -1.71, CQV 1E-11, 95th \%ile), indicating that Gpr176 is robustly and consistently downregulated during adipogenic differentiation. The SPP transcriptomic Regulation Report for Gpr176 (Supplementary Table S3 column U) contains data points documenting its regulation by prominent regulators of lipid metabolism, including FGF21, PPARG and members of the PGC-1 family. Similarly, the ChIP-Seq Regulation Report (Supplementary Table S3 column V) provides evidence for direct regulation of Gpr176 by PU.1 and numerous other nodes, including Polycomb group proteins and members of the C/EBP, STAT and BRD families. Finally, the enrichment among aPU.1KO-induced genes of HCTs for numerous characterized transcriptional regulators of circadian rhythms (Arntl/BML1, Nr1d1/Rev-Erba, Clock, and Nr3c1/ GR; Figure 5A and Supplementary Table S2) suggests that PU.1 regulation of circadian transcriptional programs in adipocytes may well extend beyond Gpr176. Set in the context of existing evidence documenting circadian connections between adipose tissue biology, lipid metabolism and the immune system (Krueger and Feldman, 2013; Lekkas and Paschos, 2019; Lananna and Musiek, 2020), the SPP data points suggest a hypothesis implicating PU.1 as a transcriptional co-ordinator of circadian programs in adipocytes and immune cells. Indeed, such a notion is supported by a previous report of global enhancement of PU.1 transactivation in Arntl/BML1-depleted macrophages (Oishi et al., 2017).

The recent characterization of PU.1 as a transcriptional driver of fibrosis (Wohlfahrt et al., 2019) is interesting given the known role of fibrosis in supporting the inflammatory state (Crewe et al., 2017) and obesity (Chiang et al., 2011). Interestingly, RPA identified a strong repression in the aPU.1KO adipocytes of pathways related to the extracellular matrix (ECM), a critical player in the development of fibrosis (Herrera et al., 2018) (Figure 4B). Inspecting the aPU.1KO-repressed genes more closely, we identified three members of the fibrinogen family $(\mathrm{OR}=318, P=1 \mathrm{E}-08$, hypergeometric test), six members of the integrin family $(\mathrm{OR}=7.5 ; \mathrm{P}=1 \mathrm{E}-04$, hypergeometric test), 12 members of the cluster of differentiation group ( $\mathrm{OR}=13, P=1 \mathrm{E}-10$, hypergeometric test) and nine members of the collagen family (OR $=6.9, \mathrm{P}=$ $5 \mathrm{E}-06$, hypergeometric test), many of which have been implicated in fibrosis and obesity (Ditschuneit et al., 1995;
Féral et al., 2008; Wynn, 2008; Khan et al., 2009; Pasarica et al., 2009; Yamauchi et al., 2011; Craciun et al., 2014; Dankel et al., 2014; Schnittert et al., 2018). Most intriguingly of all perhaps, aPU.1KO-repressed genes contain eight members of the major urinary protein (MUP) family $(\mathrm{OR}=417, P=4 \mathrm{E}-21)$, several of which are among the most strongly repressed genes. MUP proteins are related to members of the lipocalin family, which have documented connections to a variety of fibrotic conditions (Eichler et al., 1999; Ikezoe et al., 2014; Chen et al., 2020), as well as obesity and insulin resistance (Wang et al., 2007; Yan et al., 2007). Collectively, our analysis data point to a pivotal role for PU.1 in driving fibrotic transcriptional programs that support inflammatory pathways in adipocytes. Taken together, our transcriptome and bioinformatics analyzes provide valuable insights into the action of PU.1 in adipocytes. However, we need to validate these leads with a larger set of samples in follow-up studies.

Our observation that PU.1 plays a role in the development of age-associated metabolic syndrome shed light on not only a novel PU.1 action in adipocytes, but also the nature of agerelated metabolic defects. Metabolic changes developed during the aging process share similarities with that caused by obesity, but also possess some unique characteristics (Bapat et al., 2015). The underlying mechanism is not well characterized. A recent study identified a sub-population of adipocytes present only in the subcutaneous adipose tissue of older mice or humans (Nguyen et al., 2021). These cells have elevated PU.1 expression, which causes defective adipogenesis and proinflammatory cytokines secretion to inhibit adipogenesis of neighboring cells. This finding is in agreement with our results, supporting an important role of PU.1 in aging adipose tissue, in the development of age-associated adipocyte dysfunction, with a likely consequence of whole-body metabolic defects.

As an adipocyte-specific knockout, our model underscores the contribution of adipocyte-autonomous functions of PU.1 to disorders of systemic metabolism. However, PU.1 in tissues other than adipose may also contribute to metabolic syndrome. For example, expression of hepatic PU.1 is also elevated in diet-induced obese and diabetic mice, and is positively correlated with insulin resistance and liver inflammation in humans (Liu et al., 2020). Depletion of PU.1 in non-parenchymal liver cells, likely in liver macrophages, inhibited liver inflammation, hepatic steatosis and whole body insulin resistance (Liu et al., 2020). Taken together, PU.1 regulates metabolic functions in both adipocytes and in liver macrophages. Therefore, PU.1 is an important driver for metabolic disorders when animals get older and may serve as a therapeutic target for the treatment of metabolic syndrome.

\section{DATA AVAILABILITY STATEMENT}

The datasets presented in this study can be found in online repositories. The names of the repository/repositories and accession number(s) can be found below: https://www.ncbi. nlm.nih.gov/geo/query/acc.cgi?acc=GSE188497. 


\section{ETHICS STATEMENT}

The animal study was reviewed and approved by the Institution of Animal Care and Use Committee at Baylor College of Medicine.

\section{AUTHOR CONTRIBUTIONS}

Conceptualization: QT Investigation: $\mathrm{KC}, \mathrm{AD}, \mathrm{XG}$, and $\mathrm{WP}$. Validation: EL and DS. Methodology/Software: SO and NM. Formal analysis: AM, SO, and NM. Writing and editing: QT, AM, YS, and NM. Funding acquisition: QT and NM.

\section{FUNDING}

This work was supported by a US Department of Agriculture grant (3092-5-001-059), NIH (DK075978) and AHA

\section{REFERENCES}

Ağaç, D., Estrada, L. D., Maples, R., Hooper, L. V., and Farrar, J. D. (2018). The $\beta 2$ adrenergic Receptor Controls Inflammation by Driving Rapid IL-10 Secretion. Brain Behav. Immun. 74, 176-185. doi:10.1016/j.bbi.2018.09.004

Bapat, S. P., Myoung Suh, J., Fang, S., Liu, S., Zhang, Y., Cheng, A., et al. (2015). Depletion of Fat-Resident Treg Cells Prevents Age-Associated Insulin Resistance. Nature 528, 137-141. doi:10.1038/nature16151

Carotta, S., Dakic, A., D’Amico, A., Pang, S. H. M., Greig, K. T., Nutt, S. L., et al. (2010). The Transcription Factor PU.1 Controls Dendritic Cell Development and Flt3 Cytokine Receptor Expression in a Dose-dependent Manner. Immunity 32, 628-641. doi:10.1016/j.immuni.2010.05.005

Chen, J., Argemi, J., Odena, G., Xu, M.-J., Cai, Y., Massey, V., et al. (2020). Hepatic Lipocalin 2 Promotes Liver Fibrosis and portal Hypertension. Sci. Rep. 10, 15558. doi:10.1038/s41598-020-72172-7

Chiang, D. J., Pritchard, M. T., and Nagy, L. E. (2011). Obesity, Diabetes Mellitus, and Liver Fibrosis. Am. J. Physiology-Gastrointestinal Liver Physiol. 300, G697-G702. doi:10.1152/ajpgi.00426.2010

Chun, T.-H., Inoue, M., Morisaki, H., Yamanaka, I., Miyamoto, Y., Okamura, T., et al. (2010). Genetic Link between Obesity andMMP14-dependent Adipogenic Collagen Turnover. Diabetes 59, 2484-2494. doi:10.2337/db10-0073

Chung, K.-J., Chatzigeorgiou, A., Economopoulou, M., Garcia-Martin, R., Alexaki, V. I., Mitroulis, I., et al. (2017). A Self-Sustained Loop of Inflammation-Driven Inhibition of Beige Adipogenesis in Obesity. Nat. Immunol. 18, 654-664. doi:10.1038/ni.3728

Collins, S., and Surwit, R. S. (2001). The -Adrenergic Receptors and the Control of Adipose Tissue Metabolism and Thermogenesis. Recent Prog. Horm. Res. 56, 309-328. doi:10.1210/rp.56.1.309

Craciun, F. L., Ajay, A. K., Hoffmann, D., Saikumar, J., Fabian, S. L., Bijol, V., et al. (2014). Pharmacological and Genetic Depletion of Fibrinogen Protects from Kidney Fibrosis. Am. J. Physiology-Renal Physiol. 307, F471-F484. doi:10.1152/ ajprenal.00189.2014

Crewe, C., An, Y. A., and Scherer, P. E. (2017). The Ominous Triad of Adipose Tissue Dysfunction: Inflammation, Fibrosis, and Impaired Angiogenesis. J. Clin. Invest. 127, 74-82. doi:10.1172/jci88883

Dankel, S. N., Svärd, J., Matthä, S., Claussnitzer, M., Klöting, N., Glunk, V., et al. (2014). COL6A3 Expression in Adipocytes Associates with Insulin Resistance and Depends on PPAR $\gamma$ and Adipocyte Size. Obesity 22, 1807-1813. doi:10.1002/oby.20758

Debruyne, J. P. (2008). Oscillating Perceptions: the Ups and downs of the CLOCK Protein in the Mouse Circadian System. J. Genet. 87, 437-446. doi:10.1007/ s12041-008-0066-7
(18TPA34170539) to QT, by NIH DK097748 to NM, and by the DKNET Summer of Data student internship, supported by DK097748 to AM. These sponsors play no role in study design; in data acquisition, analysis and interpretation; in the writing of the manuscript; and in the decision to submit for publication.

\section{ACKNOWLEDGMENTS}

We would also like to thank Marta Fiorotto and Firoz Vohra for help with the measurement of energy expenditure of mice.

\section{SUPPLEMENTARY MATERIAL}

The Supplementary Material for this article can be found online at: https://www.frontiersin.org/articles/10.3389/fragi.2021.803482/ full\#supplementary-material

Deng, T., Lyon, C. J., Minze, L. J., Lin, J., Zou, J., Liu, J. Z., et al. (2013). Class II Major Histocompatibility Complex Plays an Essential Role in Obesity-Induced Adipose Inflammation. Cel. Metab. 17, 411-422. doi:10.1016/j.cmet.2013.02.009 DiSpirito, J. R., Fang, B., Wang, F., and Lazar, M. A. (2013). Pruning of the Adipocyte Peroxisome Proliferator-Activated Receptor $\gamma$ Cistrome by Hematopoietic Master Regulator PU.1. Mol. Cel. Biol. 33, 3354-3364. doi:10.1128/mcb.00599-13

Ditschuneit, H. H., Flechtner-Mors, M., and Adler, G. (1995). Fibrinogen in Obesity before and after Weight Reduction. Obes. Res. 3, 43-48. doi:10.1002/j.1550-8528.1995.tb00119.x

Doi, M., Murai, I., Kunisue, S., Setsu, G., Uchio, N., Tanaka, R., et al. (2016). Gpr176 Is a Gz-Linked Orphan G-Protein-Coupled Receptor that Sets the Pace of Circadian Behaviour. Nat. Commun. 7, 10583. doi:10.1038/ncomms10583

Dreyer, M. G., Juge-Aubry, C. E., Gabay, C., Lang, U., Rohner-jeanrenaud, F., Dayer, J.-M., et al. (2003). Leptin Activates the Promoter of the Interleukin-1 Receptor Antagonist through P42/44 Mitogen-Activated Protein Kinase and a Composite Nuclear Factor kappaB/PU.1 Binding Site. Biochem. J. 370, 591-599. doi:10.1042/bj20021270

Eguchi, J., Wang, X., Yu, S., Kershaw, E. E., Chiu, P. C., Dushay, J., et al. (2011). Transcriptional Control of Adipose Lipid Handling by IRF4. Cel. Metab. 13, 249-259. doi:10.1016/j.cmet.2011.02.005

Eguchi, J., Yan, Q.-W., Schones, D. E., Kamal, M., Hsu, C.-H., Zhang, M. Q., et al. (2008). Interferon Regulatory Factors Are Transcriptional Regulators of Adipogenesis. Cel. Metab. 7, 86-94. doi:10.1016/j.cmet.2007.11.002

Eichler, I., Nilsson, M., Rath, R., Enander, I., Venge, P., and Koller, D. Y. (1999). Human Neutrophil Lipocalin, a Highly Specific Marker for Acute Exacerbation in Cystic Fibrosis. Eur. Respir. J. 14, 1145-1149. doi:10.1183/ 09031936.99.14511459

Ellis, J. M., Li, L. O., Wu, P.-C., Koves, T. R., Ilkayeva, O., Stevens, R. D., et al. (2010). Adipose Acyl-CoA Synthetase-1 Directs Fatty Acids toward $\beta$ Oxidation and Is Required for Cold Thermogenesis. Cel. Metab. 12, 53-64. doi:10.1016/j.cmet.2010.05.012

Ellis, S. L., Gysbers, V., Manders, P. M., Li, W., Hofer, M. J., Müller, M., et al. (2010). The Cell-specific Induction of CXC Chemokine Ligand 9 Mediated by IFN- $\gamma$ in Microglia of the Central Nervous System Is Determined by the Myeloid Transcription Factor PU.1. J.I. 185, 1864-1877. doi:10.4049/jimmunol.1000900

Fathzadeh, M., Li, J., Rao, A., Cook, N., Chennamsetty, I., Seldin, M., et al. (2020). FAM13A Affects Body Fat Distribution and Adipocyte Function. Nat. Commun. 11, 1465. doi:10.1038/s41467-020-15291-Z

Féral, C. C., Neels, J. G., Kummer, C., Slepak, M., Olefsky, J. M., and Ginsberg, M. H. (2008). Blockade of Alpha4 Integrin Signaling Ameliorates the Metabolic Consequences of High-Fat Diet-Induced Obesity. Diabetes 57, 1842-1851. doi:10.1158/0008-5472 
Finck, B. N., Bernal-Mizrachi, C., Han, D. H., Coleman, T., Sambandam, N., LaRiviere, L. L., et al. (2005). A Potential Link between Muscle Peroxisome Proliferator- Activated Receptor- $\alpha$ Signaling and Obesity-Related Diabetes. Cel. Metab. 1, 133-144. doi:10.1016/j.cmet.2005.01.006

Gregor, M. F., and Hotamisligil, G. S. (2011). Inflammatory Mechanisms in Obesity. Annu. Rev. Immunol. 29, 415-445. doi:10.1146/annurev-immunol031210-101322

Heinz, S., Benner, C., Spann, N., Bertolino, E., Lin, Y. C., Laslo, P., et al. (2010). Simple Combinations of Lineage-Determining Transcription Factors Prime Cis-Regulatory Elements Required for Macrophage and B Cell Identities. Mol. Cel. 38, 576-589. doi:10.1016/j.molcel.2010.05.004

Herrera, J., Henke, C. A., and Bitterman, P. B. (2018). Extracellular Matrix as a Driver of Progressive Fibrosis. J. Clin. Invest. 128, 45-53. doi:10.1172/jci93557

Hotamisligil, G. S. (2017). Inflammation, Metaflammation and Immunometabolic Disorders. Nature 542, 177-185. doi:10.1038/nature21363

Hu, E., Liang, P., and Spiegelman, B. M. (1996). AdipoQ Is a Novel Adiposespecific Gene Dysregulated in Obesity. J. Biol. Chem. 271, 10697-10703. doi:10.1074/jbc.271.18.10697

Ikezoe, K., Handa, T., Mori, K., Watanabe, K., Tanizawa, K., Aihara, K., et al. (2014). Neutrophil Gelatinase-Associated Lipocalin in Idiopathic Pulmonary Fibrosis. Eur. Respir. J. 43, 1807-1809. doi:10.1183/09031936.00192613

Imai, T., Takakuwa, R., Marchand, S., Dentz, E., Bornert, J.-M., Messaddeq, N., et al. (2004). Peroxisome Proliferator-Activated Receptor Is Required in Mature white and Brown Adipocytes for Their Survival in the Mouse. Proc. Natl. Acad. Sci. 101, 4543-4547. doi:10.1073/pnas.0400356101

Iwasaki, H., Somoza, C., Shigematsu, H., Duprez, E. A., Iwasaki-Arai, J., Mizuno, S.-i., et al. (2005). Distinctive and Indispensable Roles of PU.1 in Maintenance of Hematopoietic Stem Cells and Their Differentiation. Blood 106, 1590-1600. doi:10.1182/blood-2005-03-0860

Kamei, N., Tobe, K., Suzuki, R., Ohsugi, M., Watanabe, T., Kubota, N., et al. (2006). Overexpression of Monocyte Chemoattractant Protein-1 in Adipose Tissues Causes Macrophage Recruitment and Insulin Resistance. J. Biol. Chem. 281, 26602-26614. doi:10.1074/jbc.m601284200

Karpurapu, M., Wang, X., Deng, J., Park, H., Xiao, L., Sadikot, R. T., et al. (2011). Functional PU.1 in Macrophages Has a Pivotal Role in NF-Kb Activation and Neutrophilic Lung Inflammation during Endotoxemia. Blood 118, 5255-5266. doi:10.1182/blood-2011-03-341123

Keller, P., Petrie, J. T., De Rose, P., Gerin, I., Wright, W. S., Chiang, S.-H., et al. (2008). Fat-specific Protein 27 Regulates Storage of Triacylglycerol. J. Biol. Chem. 283, 14355-14365. doi:10.1074/jbc.m708323200

Khan, T., Muise, E. S., Iyengar, P., Wang, Z. V., Chandalia, M., Abate, N., et al. (2009). Metabolic Dysregulation and Adipose Tissue Fibrosis: Role of Collagen VI. Mol. Cel. Biol. 29, 1575-1591. doi:10.1128/mcb.01300-08

Kierdorf, K., Erny, D., Goldmann, T., Sander, V., Schulz, C., Perdiguero, E. G., et al. (2013). Microglia Emerge from Erythromyeloid Precursors via Pu.1- and Irf8dependent Pathways. Nat. Neurosci. 16, 273-280. doi:10.1038/nn.3318

Kim, S.-J., Choi, Y., Choi, Y.-H., and Park, T. (2012). Obesity Activates Toll-like Receptor-Mediated Proinflammatory Signaling Cascades in the Adipose Tissue of Mice. J. Nutr. Biochem. 23, 113-122. doi:10.1016/j.jnutbio.2010.10.012

Klemsz, M. J., McKercher, S. R., Celada, A., Van Beveren, C., and Maki, R. A. (1990). The Macrophage and B Cell-specific Transcription Factor PU.1 Is Related to the Ets Oncogene. Cell 61, 113-124. doi:10.1016/0092-8674(90) 90219-5

Krueger, K. C., and Feldman, B. J. (2013). Adipose Circadian Clocks: Coordination of Metabolic Rhythms by Clock Genes, Steroid Hormones, and PPARs. Horm. Mol. Biol. Clin. Investig. 14, 15-24. doi:10.1515/hmbci-2013-0011

Kubota, N., Terauchi, Y., Miki, H., Tamemoto, H., Yamauchi, T., Komeda, K., et al. (1999). PPAR $\gamma$ Mediates High-Fat Diet-Induced Adipocyte Hypertrophy and Insulin Resistance. Mol. Cel. 4, 597-609. doi:10.1016/s1097-2765(00)80210-5

Kumari, M., Wang, X., Lantier, L., Lyubetskaya, A., Eguchi, J., Kang, S., et al. (2016). IRF3 Promotes Adipose Inflammation and Insulin Resistance and Represses browning. J. Clin. Invest. 126, 2839-2854. doi:10.1172/jci86080

Labzin, L. I., Schmidt, S. V., Masters, S. L., Beyer, M., Krebs, W., Klee, K., et al. (2015). ATF3 Is a Key Regulator of Macrophage IFN Responses. J.I. 195, 4446-4455. doi:10.4049/jimmunol.1500204

Lackey, D. E., Reis, F. C. G., Isaac, R., Zapata, R. C., El Ouarrat, D., Lee, Y. S., et al. (2019). Adipocyte PU.1 Knockout Promotes Insulin Sensitivity in HFD-Fed Obese Mice. Sci. Rep. 9, 14779. doi:10.1038/s41598-019-51196-8
Lananna, B. V., and Musiek, E. S. (2020). The Wrinkling of Time: Aging, Inflammation, Oxidative Stress, and the Circadian Clock in Neurodegeneration. Neurobiol. Dis. 139, 104832. doi:10.1016/ j.nbd.2020.104832

Law, M., and Shaw, D. R. (2018). "Mouse Genome Informatics (MGI) Is the International Resource for Information on the Laboratory Mouse," in Eukaryotic Genomic Databases: Methods and Protocols. Editor M. Kollmar (New York, NY: Springer New York), 141-161. doi:10.1007/978-1-49397737-6_7

Lefterova, M. I., Steger, D. J., Zhuo, D., Qatanani, M., Mullican, S. E., Tuteja, G., et al. (2010). Cell-Specific Determinants of Peroxisome Proliferator-Activated Receptor $\gamma$ Function in Adipocytes and Macrophages. Mol. Cel. Biol. 30, 2078-2089. doi:10.1128/mcb.01651-09

Lekkas, D., and Paschos, G. K. (2019). The Circadian Clock Control of Adipose Tissue Physiology and Metabolism. Auton. Neurosci. 219, 66-70. doi:10.1016/ j.autneu.2019.05.001

Lin, J., Handschin, C., and Spiegelman, B. M. (2005). Metabolic Control through the PGC-1 Family of Transcription Coactivators. Cel. Metab. 1, 361-370. doi:10.1016/j.cmet.2005.05.004

Lin, L., Pang, W., Chen, K., Wang, F., Gengler, J., Sun, Y., et al. (2012). Adipocyte Expression of PU.1 Transcription Factor Causes Insulin Resistance through Upregulation of Inflammatory Cytokine Gene Expression and ROS Production. Am. J. Physiology-Endocrinology Metab. 302, E1550-E1559. doi:10.1152/ ajpendo.00462.2011

Liu, J., Divoux, A., Sun, J., Zhang, J., Clément, K., Glickman, J. N., et al. (2009). Genetic Deficiency and Pharmacological Stabilization of Mast Cells Reduce Diet-Induced Obesity and Diabetes in Mice. Nat. Med. 15, 940-945. doi:10.1038/nm.1994

Liu, Q., Yu, J., Wang, L., Tang, Y., Zhou, Q., Ji, S., et al. (2020). Inhibition of PU.1 Ameliorates Metabolic Dysfunction and Non-alcoholic Steatohepatitis. J. Hepatol. 73, 361-370. doi:10.1016/j.jhep.2020.02.025

Love, M. I., Huber, W., and Anders, S. (2014). Moderated Estimation of Fold Change and Dispersion for RNA-Seq Data with DESeq2. Genome Biol. 15, 550. doi:10.1186/s13059-014-0550-8

Marecki, S., and Fenton, M. J. (2000). PU.1/Interferon Regulatory Factor Interactions; Mechanisms of Transcriptional Regulation. Cbb 33, 127-148. doi:10.1385/cbb:33:2:127

McKercher, S. R., Torbett, B. E., Anderson, K. L., Henkel, G. W., Vestal, D. J., Baribault, H., et al. (1996). Targeted Disruption of the PU.1 Gene Results in Multiple Hematopoietic Abnormalities. EMBO J. 15, 5647-5658. doi:10.1002/ j.1460-2075.1996.tb00949.x

Menet, J. S., Pescatore, S., and Rosbash, M. (2014). CLOCK:BMAL1 Is a pioneer-like Transcription Factor. Genes Dev. 28, 8-13. doi:10.1101/ gad.228536.113

Nguyen, H. P., Lin, F., Yi, D., Xie, Y., Dinh, J., Xue, P., et al. (2021). Agingdependent Regulatory Cells Emerge in Subcutaneous Fat to Inhibit Adipogenesis. Dev. Cel. 56 (10), 1437-1451. doi:10.1016/j.devcel.2021.03.026

Nguyen, V. T., and Benveniste, E. N. (2000). Involvement of STAT-1 and Ets Family Members in Interferon- $\gamma$ Induction of CD40 Transcription in Microglia/Macrophages. J. Biol. Chem. 275, 23674-23684. doi:10.1074/ jbc.m002482200

Nishimura, S., Manabe, I., Nagasaki, M., Eto, K., Yamashita, H., Ohsugi, M., et al. (2009). CD8+ Effector T Cells Contribute to Macrophage Recruitment and Adipose Tissue Inflammation in Obesity. Nat. Med. 15, 914-920. doi:10.1038/ nm.1964

Ochsner, S. A., Abraham, D., Martin, K., Ding, W., McOwiti, A., Kankanamge, W., et al. (2019). The Signaling Pathways Project, an Integrated 'omics Knowledgebase for Mammalian Cellular Signaling Pathways. Sci. Data 6, 252. doi:10.1038/s41597-019-0193-4

Ochsner, S. A., Pillich, R. T., and McKenna, N. J. (2020). Consensus Transcriptional Regulatory Networks of Coronavirus-Infected Human Cells. Sci. Data 7, 314. doi:10.1038/s41597-020-00628-6

Ohmura, K., Ishimori, N., Ohmura, Y., Tokuhara, S., Nozawa, A., Horii, S., et al. (2010). Natural Killer T Cells Are Involved in Adipose Tissues Inflammation and Glucose Intolerance in Diet-Induced Obese Mice. Atvb 30, 193-199. doi:10.1161/atvbaha.109.198614

Oishi, Y., Hayashi, S., Isagawa, T., Oshima, M., Iwama, A., Shimba, S., et al. (2017). Bmal1 Regulates Inflammatory Responses in Macrophages by Modulating 
Enhancer RNA Transcription. Sci. Rep. 7, 7086. doi:10.1038/s41598-01707100-3

Oki, S., Ohta, T., Shioi, G., Hatanaka, H., Ogasawara, O., Okuda, Y., et al. (2018). ChIP-Atlas: a Data-Mining Suite Powered by Full Integration of Public ChIPSeq Data. EMBO Rep. 19, e46255. doi:10.15252/embr.201846255

Oughtred, R., Rust, J., Chang, C., Breitkreutz, B. J., Stark, C., Willems, A., et al. (2021). TheBioGRIDdatabase: A Comprehensive Biomedical Resource of Curated Protein, Genetic, and Chemical Interactions. Protein Sci. 30, 187-200. doi:10.1002/pro.3978

Pasarica, M., Gowronska-Kozak, B., Burk, D., Remedios, I., Hymel, D., Gimble, J., et al. (2009). Adipose Tissue Collagen VI in Obesity. J. Clin. Endocrinol. Metab. 94, 5155-5162. doi:10.1210/jc.2009-0947

Patro, R., Duggal, G., Love, M. I., Irizarry, R. A., and Kingsford, C. (2017). Salmon Provides Fast and Bias-Aware Quantification of Transcript Expression. Nat. Methods 14, 417-419. doi:10.1038/nmeth.4197

Polli, M., Dakic, A., Light, A., Wu, L., Tarlinton, D. M., and Nutt, S. L. (2005). The Development of Functional B Lymphocytes in Conditional PU.1 Knock-Out Mice. Blood 106, 2083-2090. doi:10.1182/blood-2005-01-0283

Puigserver, P., Wu, Z., Park, C. W., Graves, R., Wright, M., and Spiegelman, B. M. (1998). A Cold-Inducible Coactivator of Nuclear Receptors Linked to Adaptive Thermogenesis. Cell 92, 829-839. doi:10.1016/s0092-8674(00)81410-5

Qiu, J., Zhang, Z., Wang, S., Chen, Y., Liu, C., Xu, S., et al. (2020). Transferrin Receptor Functionally Marks Thermogenic Adipocytes. Front. Cel. Dev. Biol. 8, 572459. doi:10.3389/fcell.2020.572459

Rajbhandari, P., Thomas, B. J., Feng, A.-C., Hong, C., Wang, J., Vergnes, L., et al. (2018). IL-10 Signaling Remodels Adipose Chromatin Architecture to Limit Thermogenesis and Energy Expenditure. Cell 172, 218-233. e217. doi:10.1016/ j.cell.2017.11.019

Ramakrishnan, S. N., and Muscat, G. E. (2006). The Orphan Rev-Erb Nuclear Receptors: A Link between Metabolism, Circadian Rhythm and Inflammation. Nucl. Recept. Signal. 4, e009. nrs. doi:10.1621/nrs.04009

Remeseiro, S., Cuadrado, A., Gómez-López, G., Pisano, D. G., and Losada, A. (2012). A Unique Role of Cohesin-SA1 in Gene Regulation and Development. Embo J 31, 2090-2102. doi:10.1038/emboj.2012.60

Rieske, P., and Pongubala, J. R. (2001). AKT Induces Transcriptional Activity of PU.1 through Phosphorylation-Mediated Modifications within its Transactivation Domain. J. Biol. Chem. 276, 8460-8468. doi:10.1074/ jbc.m007482200

Schnittert, J., Bansal, R., Storm, G., and Prakash, J. (2018). Integrins in Wound Healing, Fibrosis and Tumor Stroma: High Potential Targets for Therapeutics and Drug Delivery. Adv. Drug Deliv. Rev. 129, 37-53. doi:10.1016/ j.addr.2018.01.020

Scott, E. W., Simon, M. C., Anastasi, J., and Singh, H. (1994). Requirement of Transcription Factor PU.1 in the Development of Multiple Hematopoietic Lineages. Science 265, 1573-1577. doi:10.1126/science.8079170

Shimba, S., Ogawa, T., Hitosugi, S., Ichihashi, Y., Nakadaira, Y., Kobayashi, M., et al. (2011). Deficient of a Clock Gene, Brain and Muscle Arnt-like Protein-1 (BMAL1), Induces Dyslipidemia and Ectopic Fat Formation. PLoS One 6, e25231. doi:10.1371/journal.pone.0025231

Smith, C. L., and Eppig, J. T. (2009). The Mammalian Phenotype Ontology: Enabling Robust Annotation and Comparative Analysis. Wires Syst. Biol. Med. 1, 390-399. doi:10.1002/wsbm.44

So, A. Y.-L., Bernal, T. U., Pillsbury, M. L., Yamamoto, K. R., and Feldman, B. J. (2009). Glucocorticoid Regulation of the Circadian Clock Modulates Glucose Homeostasis. Proc. Natl. Acad. Sci. 106, 17582-17587. doi:10.1073/ pnas.0909733106

Steidl, U., Rosenbauer, F., Verhaak, R. G. W., Gu, X., Ebralidze, A., Otu, H. H., et al. (2006). Essential Role of Jun Family Transcription Factors in PU.1 KnockdownInduced Leukemic Stem Cells. Nat. Genet. 38, 1269-1277. doi:10.1038/ng1898

Stienstra, R., Joosten, L. A. B., Koenen, T., van Tits, B., van Diepen, J. A., van den Berg, S. A. A., et al. (2010). The Inflammasome-Mediated Caspase-1 Activation Controls Adipocyte Differentiation and Insulin Sensitivity. Cel. Metab. 12, 593-605. doi:10.1016/j.cmet.2010.11.011

Subramanian, A., Tamayo, P., Mootha, V. K., Mukherjee, S., Ebert, B. L., Gillette, M. A., et al. (2005). Gene Set Enrichment Analysis: a Knowledge-Based Approach for Interpreting Genome-wide Expression Profiles. Proc. Natl. Acad. Sci. 102, 15545-15550. doi:10.1073/pnas.0506580102
Tondravi, M. M., McKercher, S. R., Anderson, K., Erdmann, J. M., Quiroz, M., Maki, R., et al. (1997). Osteopetrosis in Mice Lacking Haematopoietic Transcription Factor PU.1. Nature 386, 81-84. doi:10.1038/386081a0

Turek, F. W., Joshu, C., Kohsaka, A., Lin, E., Ivanova, G., McDearmon, E., et al. (2005). Obesity and Metabolic Syndrome in Circadian Clock Mutant Mice. Science 308, 1043-1045. doi:10.1126/science.1108750

Wang, F., and Tong, Q. (2008). Transcription Factor PU.1 Is Expressed in white Adipose and Inhibits Adipocyte Differentiation. Am. J. Physiology-Cell Physiol. 295, C213-C220. doi:10.1152/ajpcell.00422.2007

Wang, M., Liu, Z., Liu, C., Wu, T., Cai, F., Wang, Q., et al. (2016). PU.1 Is Involved in the Immune Response to Aspergillus fumigatus through Upregulating Dectin-1 Expression. BMC Infect. Dis. 16, 297. doi:10.1186/s12879-016$1632-\mathrm{x}$

Wang, N.-d., Finegold, M. J., Bradley, A., Ou, C. N., Abdelsayed, S. V., Wilde, M. D., et al. (1995). Impaired Energy Homeostasis in C/EBPa Knockout Mice. Science 269, 1108-1112. doi:10.1126/science.7652557

Wang, Y., Lam, K. S. L., Kraegen, E. W., Sweeney, G., Zhang, J., Tso, A. W., et al. (2007). Lipocalin-2 Is an Inflammatory Marker Closely Associated with Obesity, Insulin Resistance, and Hyperglycemia in Humans. Clin. Chem. 53, 34-41. doi:10.1373/clinchem.2006.075614

Weisberg, S. P., Hunter, D., Huber, R., Lemieux, J., Slaymaker, S., Vaddi, K., et al. (2006). CCR2 Modulates Inflammatory and Metabolic Effects of High-Fat Feeding. J. Clin. Invest. 116, 115-124. doi:10.1172/jci24335

Weisberg, S. P., McCann, D., Desai, M., Rosenbaum, M., Leibel, R. L., and Ferrante, A. W. (2003). Obesity Is Associated with Macrophage Accumulation in Adipose Tissue. J. Clin. Invest. 112, 1796-1808. doi:10.1172/jci200319246

Winer, S., Chan, Y., Paltser, G., Truong, D., Tsui, H., Bahrami, J., et al. (2009). Normalization of Obesity-Associated Insulin Resistance through Immunotherapy. Nat. Med. 15, 921-929. doi:10.1038/nm.2001

Wohlfahrt, T., Rauber, S., Uebe, S., Luber, M., Soare, A., Ekici, A., et al. (2019). PU.1 Controls Fibroblast Polarization and Tissue Fibrosis. Nature 566, 344-349. doi:10.1038/s41586-019-0896-x

Wynn, T. (2008). Cellular and Molecular Mechanisms of Fibrosis. J. Pathol. 214, 199-210. doi:10.1002/path.2277

Xu, H., Barnes, G. T., Yang, Q., Tan, G., Yang, D., Chou, C. J., et al. (2003). Chronic Inflammation in Fat Plays a Crucial Role in the Development of ObesityRelated Insulin Resistance. J. Clin. Invest. 112, 1821-1830. doi:10.1172/ jci200319451

Yamauchi, K., Kasuya, Y., Kuroda, F., Tanaka, K., Tsuyusaki, J., Ishizaki, S., et al. (2011). Attenuation of Lung Inflammation and Fibrosis in CD69-Deficient Mice after Intratracheal Bleomycin. Respir. Res. 12, 131. doi:10.1186/14659921-12-131

Yan, Q.-W., Yang, Q., Mody, N., Graham, T. E., Hsu, C.-H., Xu, Z., et al. (2007). The Adipokine Lipocalin 2 Is Regulated by Obesity and Promotes Insulin Resistance. Diabetes 56, 2533-2540. doi:10.2337/ db07-0007

Zhou, H., Wan, B., Grubisic, I., Kaplan, T., and Tjian, R. (2014). TAF7L Modulates Brown Adipose Tissue Formation. Elife 3, e02811. doi:10.7554/eLife.02811

Conflict of Interest: The authors declare that the research was conducted in the absence of any commercial or financial relationships that could be construed as a potential conflict of interest.

Publisher's Note: All claims expressed in this article are solely those of the authors and do not necessarily represent those of their affiliated organizations, or those of the publisher, the editors and the reviewers. Any product that may be evaluated in this article, or claim that may be made by its manufacturer, is not guaranteed or endorsed by the publisher.

Copyright $\odot 2022$ Chen, De Angulo, Guo, More, Ochsner, Lopez, Saul, Pang, Sun, McKenna and Tong. This is an open-access article distributed under the terms of the Creative Commons Attribution License (CC BY). The use, distribution or reproduction in other forums is permitted, provided the original author(s) and the copyright owner(s) are credited and that the original publication in this journal is cited, in accordance with accepted academic practice. No use, distribution or reproduction is permitted which does not comply with these terms. 